\title{
Thermodynamics of the Phase Equilibriums of Some Organic Compounds
}

\author{
Raisa Varushchenko and Anna Druzhinina \\ Lomonosov Moscow State University \\ Russia
}

\section{Introduction}

A comprehensive investigation of the phase equilibriums and determination of thermodynamic properties of pure substances is a significant object of the chemical thermodynamics. Data on the phase transitions, heat capacities, and saturation vapor pressure over the solid and liquid phases are used in many fields of science and technology, including calculations on the basis of the third law of thermodynamics. Theoretical and practical applications of thermodynamic data require verification of their reliability. The Clapeyron equation combines different properties of coexisting phases: temperature, vapor pressure, volume, enthalpy of the phase transitions, and caloric values $C_{p}$ and $C_{v}$. Using this equation allows one to verify numerical data for thermodynamic concordance, to reveal unreliable quantities, and to predict failing thermodynamic properties. Mutual concordance and reliability of the calorimetric data on the heat capacity, the saturated vapor pressures, and the properties of phase transition can be verified by comparison of the absolute entropies determined from the experimental data by the third thermodynamic law, $S_{m}^{O}(g)($ expt $)$ with those ones calculated by statistical thermodynamics, $S_{m}^{O}($ stat $)$. A congruence of these values within errors limits justifies their reliability. Critical analyses of the recent data on thermodynamic properties of some organic compounds are published by the National Institute of the Standards and Technology [NIST], USA. Literature data on the vapor pressures and the enthalpies of vaporization for $n$-alkanes $C_{5}-C_{20}$ were reviewed and critically analyzed in the reference (Ruzicka \& Majer, 1994). Thermodynamic properties of many classes of organic compounds were considered in monograph (Domalski \& Hearing, 1993; Poling et al., 2001) that favoured the development of the Benson's calculation method.

This chapter deals with reviewing and summarizing the data on the phase equilibriums carried out for some functional organic compounds by the low temperature adiabatic calorimetry, comparative ebulliometry, and vaporization calorimetry in the Luginin's Laboratory of Thermochemistry [LLT] of the Moscow State University [MSU] and other research centres. The numerous data on the heat capacity, the vapor pressure, enthalpies of the phase transitions, and derived thermodynamic functions were obtained for series of freons, cyclic hydrocarbons and fluorocarbons, and derivatives of ferrocene. A sufficient attention was given to the critical analyses of the thermodynamic data, their reliability, and to interconnections between the properties and some structural parameters of the 
compounds. Experimental and calculation methods for determination of the properties rely mostly on the LLT-school.

Freons are halogen derivatives of ethane and propane which possess a unique combination of the useful properties: high volatility, high enthalpy of vaporization, no combustibility, biological inertness, etc. Due to these properties, freons have found a wide application in many areas of science, technology, and medicine (Varushchenko et. al., 2007).

Alkyl derivatives of adamantine, $\mathrm{C}_{10} \mathrm{H}_{16}, \square$, are of an interest due to tendency to form plastic crystals. Bicyclical cis- and trans- isomers of decaline, $\mathrm{C}_{10} \mathrm{H}_{18}, \bigcirc$, and hydrindane, $\mathrm{C}_{9} \mathrm{H}_{16}, \bigcirc$, have poor intermolecular interactions and also form plastic crystals. Their perfluoride counterparts exhibit high chemical stability, absolute biological inertness, and capacity for dissolving and transferring large amounts of gases, in particular, oxygen and carbon dioxide. Due to these properties, perfluorocarbons have found wide application in biology and medicine as effective gas-transferring media and artificial blood substitutes. A mixture of perfluorodecaline, $\mathrm{C}_{10} \mathrm{~F}_{18}$, and perfluoro-N-(4-methylcyclohexyl)piperidine, $\mathrm{C}_{12} \mathrm{~F}_{23} \mathrm{~N}$, forms of the "Ftorosan" blood substitute (Russia) (Ries, 1991). Bicyclical cis- and trans- isomers of decaline and hydrindane are of the interest in study of an interconnection between thermodynamic properties and the structure of the compounds when passing from perfluorocarbons to their hydrocarbon counterparts.

Alkyl- and acyl- ferrocene derivatives [FD] are the sandwich-type organometallic compounds discovered in the 50th years of the $\mathrm{XX}$ century. Owing to favourable conjunction of the chemical and physical properties, namely low toxicity, high thermal stability, and volatility, some FD has found ever-increasing application in technology (electric materials, regulators of fuel combustion etc.) and medicine (anti-cancer and blood-creating drugs).

This chapter is intended for researchers with an interest in measuring characteristics of the phase transitions and in determination of the equilibrium properties by experimental and theoretical methods. A number of relationships for practical use are represented with illustrative examples and necessary recommendations. The chapter contains main references to the literature used in reviewing and summarizing the numerous data on the properties of some functional organic compounds.

Part 2 deals with the ebulliometric and transpiration methods for determination of the saturation vapor pressure in dependence on the temperature. Design of devices and experimental techniques and mathematical processing of the vapor pressures are given. A modified ebulliometer of an original construction was given for determination of the $p T$ parameters in moderate ("atmospheric") pressure region. The enthalpies of vaporization obtained by direct calorimetric method and those ones calculated from the vapor pressure are compared for justifying their reliability. An interconnection between the properties derived from the vapor pressure and some structural parameters of the substances are analyzed.

Part 3 considers the low-temperature adiabatic calorimetry for measuring the heat capacity and studying the properties of the phase transitions. Experimental technique has been presented by modern completely automated adiabatic calorimeter used in LLT. Experimental determination and mathematical processing of the phase transitions were given including an X-ray analysis of crystal structure and the infrared and Raman spectroscopy for interpretation of the processes occurring during the solid-phase transitions. Main thermodynamic functions (changes of the entropy, enthalpy, and Gibb's energy) in 
condensed states were calculated on the basis of the heat capacities and the properties of the solid-to-solid transitions and fusion.

Part 4 deals with 1) determination of the ideal gas thermodynamic functions by experimental and theoretical methods, 2) verification of the thermodynamic functions by comparing the absolute entropies calculated on the basis of the third thermodynamic law and by statistical thermodynamics, and 3) the methods of extending the saturated vapor pressure of the "atmospheric" range of pressure to entire region of liquids from the triple to the critical temperatures.

Parts 5, 6, 7, and 8 present Conclusion, Acknowledgments, References, and Appendix, respectively.

\section{Temperature dependence of saturated vapor pressure}

The values of the vapor pressure of liquid substances are mostly determined by the static and dynamic (mainly ebulliometric) methods. A comparative ebulliometry is frequently employed due to its simpler technique and suitability for the series of determinations. The greatest number of saturated vapor pressure of organic compounds was obtained by this method in the moderate ("atmospheric") range of pressure $2-3 \leq(\Delta p, \mathrm{kPa}) \leq 100-150$. The highest accurate of vapor pressure is usually attained in this range that makes it possible to obtain reliable derivative values, in particular, the enthalpies of vaporization. Few $p T$ data are available in the literature for the entire region of liquid phase because of methodical difficulties and high errors of determination at low $(<1 \mathrm{kPa})$ and high $(>200 \mathrm{kPa})$ pressures.

\subsection{Experimental and mathematical processing}

Fig. 1 presents a schematic view of a setup designed for determinations of the temperature dependence of saturation vapor pressure by comparative ebulliometry (Varouchtchenko \& Droujinina, 1995).

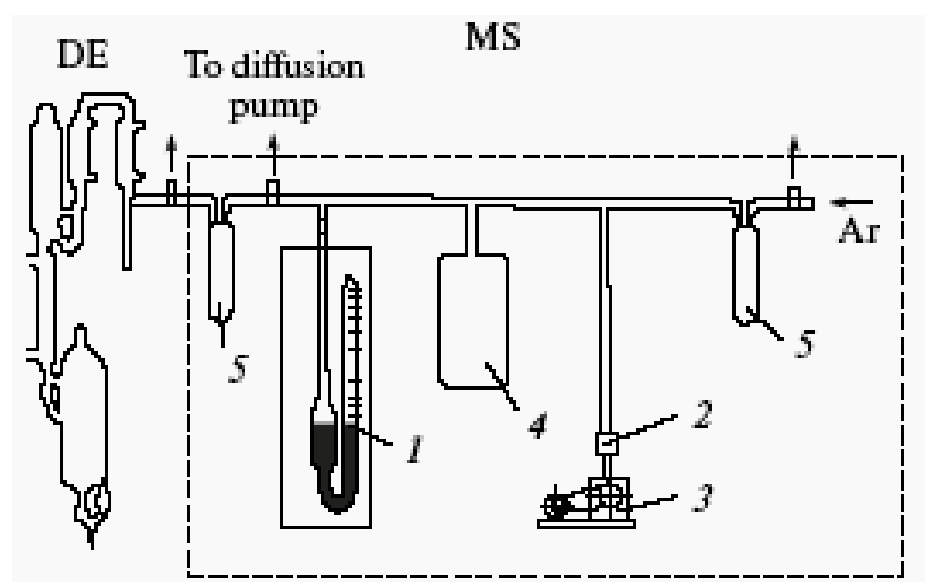

Fig. 1. The setup for determination of the $p T$ parameters: DE, differential ebulliometer; MS, manometer system; (1) mercury-contact manometer; (2) electromagnetic valve; (3) roughing pump; (4) ballast reservoir; (5) traps 


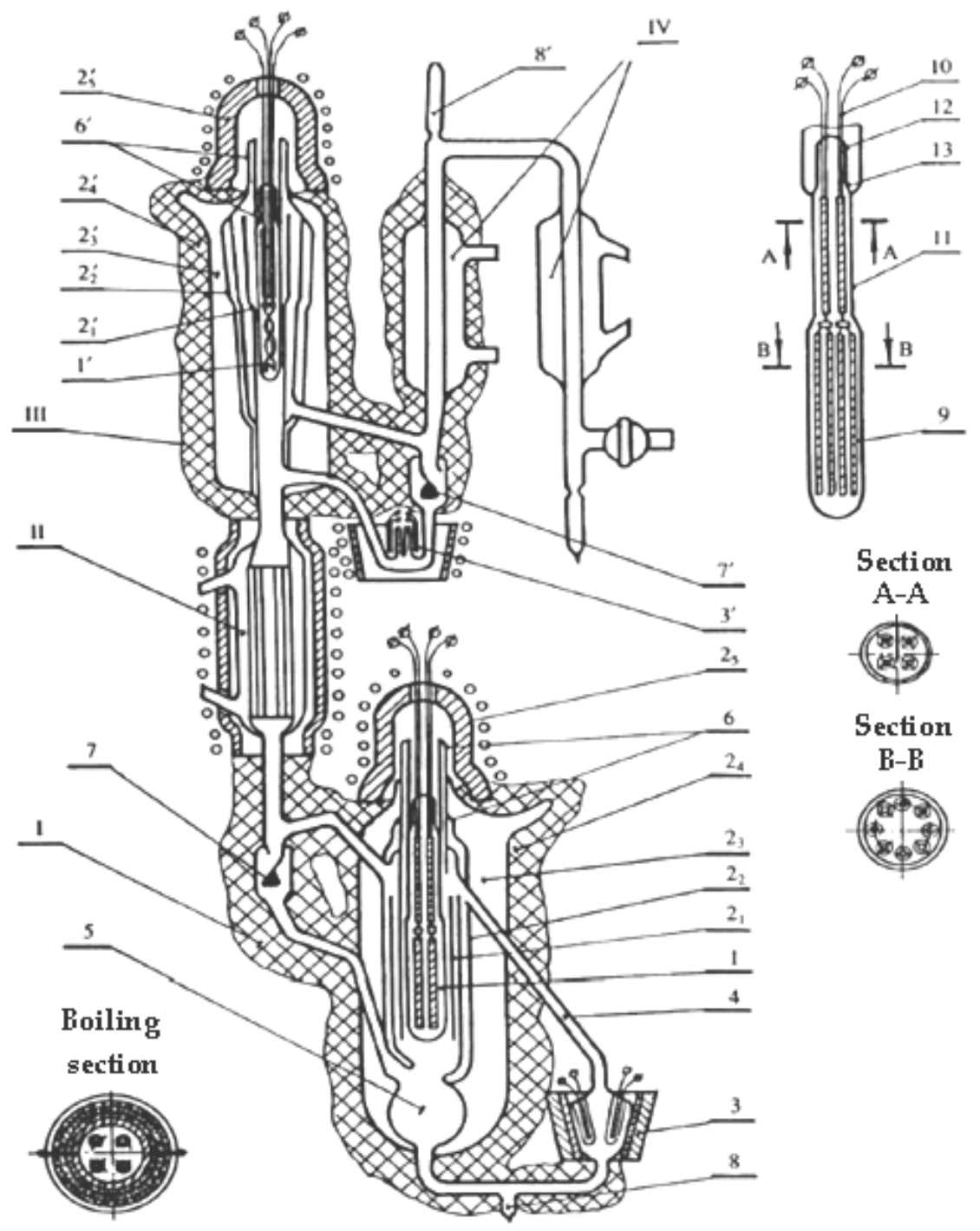

Fig. 2. The differential ebulliometer: I, boiling section; II, rectification column; III, condensation section; IV, system of coolers for returning and collecting a condensate; $\left(1,1^{\prime}\right)$ platinum resistance thermometers; $\left(2{ }_{1}, 2_{1}{ }^{\prime}\right)$ glass screens of thermometers; $\left(22_{2}, 2_{2}{ }^{\prime}\right)$ silver radiation screens; $\left(22_{3}, 2_{3}{ }^{\prime}\right)$ vacuum shells; $\left(24,2{ }^{\prime}\right)$ heat-insulating layer (asbestos); $\left(25,25^{\prime}\right)$ shells for heating the thermometer parts extending from the ebulliometer; (3) boiler; $\left(3^{\prime}\right) \mathrm{U}$ shaped liquid valve; (4) Cottrell pump; (5) spherical reservoir; (6 (13), 6') differential Chromel-Alumel thermocouples; $\left(7,7^{\prime}\right)$ droplet counters; $\left(8,8^{\prime}\right)$ branches for outlet and inlet of liquid; (9) sensing element of platinum resistance thermometer; (10) platinum wires; (11) protective glass tube; (12) Pyrex-tungsten glass-molybdenum glass transition. 
The setup consists of a differential ebulliometer used for measuring the boiling and condensation temperatures and manometer system operating in the manostat mode. The main part of MS system is a mercury-contact (tungsten) manometer that serves for automatic control and determination of the pressure inside the ebulliometer. Argon was introduced into the system to maintain the constant pressure equal to that of the saturation vapors of the substance under study. The temperature of the (liquid + vapor) equilibrium was measured at 20 fixed pressures controlled by manometer system.

A schematic view of modified Swietoslawski -type ebulliometer is given in Fig. 2. The differential ebulliometer was used for determination of the temperature dependence of the vapor pressure by measuring the boiling, $T_{\text {boil }}$, or (rarely) condensation, $T_{\text {cond }}$ ' temperatures and for estimation of an ebulliometric degree of purity for the samples by the difference $\left(T_{\text {boil }}-T_{\text {cond }}\right)$. The latter is below $0.005 \mathrm{~K}$ for the pure substances. The boiling and condensation sections and other parts of differential ebulliometer were made of "Pyrex" glass and were sealed together. The modification of the ebulliometer was directed for solving three basic problems: 1) increasing the thermometric sensitivity of a system used for temperature measurements of the (liquid - vapor) equilibrium; 2) decreasing a heat exchange of the temperature sensors with the surrounding, and 3) reducing the superheating of the boiling liquid, that leads to increasing the accuracy of the temperature measurements.

For increasing the sensitivity of the thermometers, their protecting tubes were soldered in the boiling and condensation sections of the ebulliometer. Sensing elements of vibration resistant thermometers $(R \approx 100 \Omega)$ consisted of a few platinum spirals wound around glass capillaries. The latter had coefficients of linear expiation close to that of platinum.

Connecting wires (current and potential) of the thermometer were vacuum - tight sealed through the glass-molybdenum part of a passage (12, Fig. 2) of the protecting tube. The thermometers were graduated in Mendeleev's Institute of Metrology (S. Petersburg) at the triple - point temperature of water $(273.16 \mathrm{~K})$ and melting temperatures of tin $(505.118 \mathrm{~K})$ and gallium $(302.920 \mathrm{~K})$. The summary error of graduation is $\pm 3 \cdot 10^{-3} \mathrm{~K}$. A special system of heat insulation of the thermometers was employed. It consisted of: glass screens washed by boiling liquid or by condensate $\left(2_{1}\right.$ or $\left.21^{\prime}\right)$, respectively (Fig. 2$)$; silver radiation screens; vacuum jackets ( $\left.p \approx 1.3 \cdot 10^{-3} \mathrm{~Pa}\right)$; heat - insulating layers (asbestos), and electrically heated screens $\left(25\right.$ and $\left.25^{\prime}\right)$ for upper parts of thermometers overhanging from ebulliometer. Application of such heat - insulating system made it possible to conduct precision temperature measurements without heating the main part of the ebulliometer. The error of temperature measurements caused by heat exchange of the thermometers with the surroundings, were estimated on the basis of heat - exchange laws to be $\approx 1 \cdot 10^{-3} \mathrm{~K}$. The superheating of the liquid was reduced by using several internal and two external boiler heaters which promoted to smooth boiling of the liquid. Performed modification of the ebulliometer allowed cutting down substantially the amount of liquid which was spent for heating the inner surfaces of instrument up to working temperature.

Thus, the necessary volume of liquid was reduced several times: down to $\approx 5 \mathrm{~cm}^{3}$ when measuring only the boiling temperature and to $\approx 8 \mathrm{~cm}^{3}$ when both boiling and condensation temperatures were measured. The use of the comparison method makes it possible to 
reduce the $p T$ parameters determination to the precision temperature measurements. The temperature was automatically measured by potentiometer method and the results were displayed on a personal computer [PC] screen with the aid of the AK-6.25 computermeasurement system designed at All-Russia Research Institute of Physico-technical and Radio-technical Measurements [VNIIFTRI].

An automatic maintenance of the constant pressure was attained by a mercury-contact manometer which was controlled by vacuum pump via an electromagnetic valve (Fig. 1). The pressure of argon fluctuated in the limits from $( \pm 20$ to \pm 40$) \mathrm{Pa}$. The boiling temperature was measured at the highest pressure in the cycle at the moment of mercury-to-tungsten contact. The manometer was thermostated at the temperature $(300.00 \pm 0.02) \mathrm{K}$. The measurements of the boiling and condensation temperatures were conducted after attaining thermodynamic equilibrium in the ebulliometer. To be assured that the liquid under study had not decomposed, the boiling temperature at one of initial points of the $p T$ curve was measured several times during the ebulliometric experiments.

Errors of temperature $S_{T}$ and vapor pressure $S_{p}$ measurements were calculated as:

$$
\begin{gathered}
\left|S_{T}\right|=\left\{\left(\mathrm{t} \cdot S_{\mathrm{T} 1}\right)^{2}+\left(\mathrm{S}_{\mathrm{T} 2}\right)^{2}\right\}^{1 / 2} \\
\left|S_{p}\right|=\left\{(\mathrm{d} p / \mathrm{d} T)_{1}^{2} \cdot\left(t \cdot S_{\mathrm{T} 1}\right)^{2}+(\mathrm{d} p / \mathrm{d} T)_{3}^{2} \cdot\left(t \cdot S_{\mathrm{T} 3}\right)^{2}\right\}^{1 / 2}
\end{gathered}
$$

where $\left|t \cdot S_{T 1}\right|$ and $\left|t \cdot S_{T 3}\right|$ denote the instrumental errors of the temperature measurements $\left(\leq 5 \cdot 10^{-3} K\right)$ at the substance research and at graduation of the mercury-contact manometer; $t$ is Student's criterion; $\left|S_{T 2}\right|=3 \cdot 10^{-3} K$ denotes the error of graduation of the thermometer; and $(d p / d T)_{1}$ and $(d p / d T)_{3}$ are temperature coefficients of the pressure for standard and studied substances, respectively. The total uncertainty of temperature measurement was $\left|S_{T}\right| \leq 6 \cdot 10^{-3} \mathrm{~K}$. The error of graduation of the mercury-contact manometer by means of water and $n$-decane and the error of determination of the vapor pressure of the substance under study were equal to $\mid S_{p}$ (grad.) $) \mid=\left(13\right.$ to 20) Pa and $\left|S_{p}\right|=(20$ to 26) Pa, respectively.

The accuracy of ebulliometric measurements was checked by determinations of the saturation vapor pressures of substances having significantly different boiling temperatures, namely benzene and undecane. The normal boiling temperatures of the standard substances obtained in this work agree within errors limits $\pm \leq 0.01 \mathrm{~K}$ with precise values of reference (Boublik et al., 1984).

Comparative ebulliometry was employed for determination a series of saturation vapor pressures in dependence on temperature for some freons; halogen - ethanes and -propanes; alkyladamantanes; cis- and trans- hydrindanes, cis- and trans- decalines, and their fluoridated counterparts.

The mathematical processing of the observed boiling temperatures and vapor pressures were conducted by the semi -empirical equation: 


$$
\begin{aligned}
& -R \cdot T \cdot \ln (p)=\Delta H_{m}(\langle T\rangle)-a_{1} \cdot T+a_{2} \cdot\{T-\langle T\rangle-T \cdot \ln (T /\langle T\rangle)\} \\
& -a_{3} \cdot\left\{(1 / 2)\left(T^{2}-\langle T\rangle^{2}\right)-T \cdot\langle T\rangle \cdot \ln (T /\langle T\rangle)\right\}
\end{aligned}
$$

where $\langle T\rangle$ denotes the mean temperature and $\Delta H_{m}(\langle T\rangle), \alpha_{1}, \alpha_{2}$, and $\alpha_{3}$ are parameters. Equation (1) was derived by integration of the Clapeyron equation:

$$
d \ln (p) / d T=\Delta_{\text {vap }} H_{m} /\left(\Delta Z \cdot R \cdot T^{2}\right)
$$

with the approximation for $\Delta_{v a p} H_{m} / \Delta \mathrm{Z}$ :

$$
\Delta_{\text {vap }} H_{m} / \Delta Z=\Delta H_{m}^{\prime}(T)=\Delta H_{m}(\langle T\rangle)+\alpha_{2} \cdot(T-\langle T\rangle)+(1 / 2) \cdot \alpha_{3} \cdot(T-\langle T\rangle)^{2},
$$

where $\Delta Z$ denotes the difference of compression factors of gas and liquid. Equation (3) in turn was developed by integration of the approximation for $\Delta C_{p, m}=C_{p, m}^{O}(g)-C_{p, m}($ liq $) \approx \alpha_{2}+\alpha_{3} \cdot(T-\langle T\rangle)$, as a linear function of the temperature. The treatment of the $p T$ parameters was carried out by the least-squares method [LSM] using orthogonal functions (Kornilov \& Vidavski, 1969). Mathematical processing of the saturation vapor pressures is given in Appendix. A system of normal equations of LSM is a diagonal matrix relative to the orthogonal functions. The latter are mutually independent that allows to evaluate their uncertainties and those ones for the $\ln \{p(T) / \mathrm{Pa}\}$ and $\Delta_{\text {vap }} H_{m}(T)$ functions and, as a result, to choice of an adequate number of terms of relations (1) and (3) by curtailing or expanding terms to suit the accuracy of the parameters of these relations without a new treatment of $p T$ data. Final equations for these functions are set out for compactness, as:

$$
\begin{gathered}
\ln (p / P a)=A+B / T+C \cdot \ln (T / K)+D \cdot T \\
\Delta_{\text {vap }} H_{m}=R \cdot\left(-B+C \cdot T+D \cdot T^{2}\right) \cdot \Delta Z \pm\left[s\left\{\Delta H_{m}^{\prime}(T)\right\}+\Delta_{\text {vap }} H_{m} \cdot \Delta(\Delta Z)\right]
\end{gathered}
$$

where $A, B, C$, and $D$ are constants related to the parameters of equation (1) by linear correlations; $s\left\{\Delta H_{m}^{\prime}(T)\right\}$ is the uncertainty of $\Delta_{v a p} H_{m}$ value resulting from errors of $(p, T)$ parameters; and $\Delta(\Delta Z)$ is the error of the $\Delta Z$ difference estimation. The $s\left\{\Delta H_{m}^{\prime}(T)\right\}$ values are evaluated by the law of random errors accumulation on the basis of dispersions of the orthogonal functions (Appendix).

Because the coefficients of equations (4) and (5) are correlated, the numbers of digits in $A, B$, $C$, and $D$ coefficients were selected so that the calculated $p$ values would not exceed the experimental errors of the vapor pressure determination. (Appendix). Statistical analysis of the error of the smallest parameter $\alpha_{3}$ of the equation (1) (accordingly, D of equations (4) and (5)) was evaluated by the Fisher criterion, $F$. If the inequality:

$$
F=\alpha_{3}^{2} / s^{2}\left(\alpha_{3}\right) \geq F_{0.05}(1, f),
$$




\begin{tabular}{|c|c|c|c|c|c|c|c|c|}
\hline Compounds & $\begin{array}{l}\text { Purity, } \\
\text { mol. \% }\end{array}$ & $\begin{array}{c}\Delta T(p T), \\
\mathrm{K}\end{array}$ & $n$ & A & $-B$ & $-C$ & $D \cdot 10^{3}$ & $\begin{array}{l}S_{p} \\
\mathrm{~Pa}\end{array}$ \\
\hline \multicolumn{9}{|c|}{ Freons and halogen -ethanes and -propanes } \\
\hline $\mathrm{CFCl}_{2} \mathrm{CFCl}_{2}$ & $99.30^{a}$ & $313-361$ & 12 & 59.2013 & 5922.1 & 6.71105 & 3.3075 & 2.1 \\
\hline $\mathrm{CF}_{2} \mathrm{ClCFCl}_{2}$ & $99.80^{a}$ & $298-316$ & 7 & 42.1123 & 4680.0 & 3.96897 & - & 19.0 \\
\hline $\mathrm{CF}_{2} \mathrm{ClCF}_{2} \mathrm{Cl}$ & $99.79 a$ & $178-277$ & 10 & 320.3557 & 10029.4 & 55.11366 & 110.0486 & 34.5 \\
\hline $\mathrm{CF}_{2} \mathrm{BrCF}_{2} \mathrm{Br}$ & $99.50^{c}$ & $298-320$ & 8 & 43.4680 & 4732.1 & 4.17343 & - & 10.5 \\
\hline $\mathrm{CF}_{2} \mathrm{ClCHCl}_{2}$ & $99.64^{b}$ & $297-345$ & 14 & 141.7579 & 7982.7 & 21.01696 & 25.5415 & 15.0 \\
\hline $\mathrm{CFCl}_{2} \mathrm{CHFCl}$ & $99.38^{b}$ & $289-346$ & 16 & 137.9719 & 7912.3 & 20.33885 & 24.3167 & 11.0 \\
\hline $\mathrm{CF}_{3} \mathrm{CHCl}_{2}$ & $99.83^{a}$ & $256-454$ & 45 & 90.4773 & 5766.1 & 12.41706 & 13.8312 & 3.8 \\
\hline $\mathrm{CF}_{2} \mathrm{ClCHFCl}$ & $99.51^{a}$ & $278-303$ & 9 & 655.6005 & 20714.9 & 111.4046 & 177.9514 & 10.0 \\
\hline $\mathrm{CF}_{3} \mathrm{CHClBr}$ & $99.7^{a}$ & $297-323$ & 8 & 45.2236 & 4950.1 & 4.37789 & - & 5.6 \\
\hline $\mathrm{CF}_{3} \mathrm{CH}_{2} \mathrm{CH}_{2} \mathrm{Cl}$ & $99.90^{a}$ & $297-315$ & 8 & 47.6041 & 5040.0 & 4.71465 & - & 11.9 \\
\hline $\mathrm{CF}_{3} \mathrm{CH}_{2} \mathrm{CHCl}_{2}$ & $99.30^{c}$ & $302-341$ & 12 & 133.0670 & 8026.1 & 19.24539 & 21.0475 & 13.9 \\
\hline $\mathrm{CF}_{3} \mathrm{CH}_{2} \mathrm{CCl}_{3}$ & $99.59^{a}$ & $321-364$ & 12 & 243.2798 & 11578.6 & 38.06825 & 48.1087 & 20.3 \\
\hline $\mathrm{CF}_{3} \mathrm{CH}_{2} \mathrm{CFCl}_{2}$ & $99.9^{b}$ & $297-333$ & 9 & 1415.3662 & 43459.2 & 241.45741 & 366.8286 & 36.8 \\
\hline $\mathrm{CHCl}_{2} \mathrm{CH}_{3}$ & $99.9^{b}$ & $294-330$ & 11 & 135.6402 & 7511.9 & 20.10746 & 25.2571 & 10.1 \\
\hline $\mathrm{CH}_{2} \mathrm{ClCH}_{2} \mathrm{Cl}$ & $99.9^{b}$ & 299-356 & 15 & 83.3156 & 6652.5 & 10.77546 & 9.2008 & 6.2 \\
\hline $\mathrm{CH}_{2} \mathrm{BrCH}_{2} \mathrm{Br}$ & $99.9^{b}$ & $331-426$ & 12 & 127.1428 & 8810.4 & 17.99911 & 18.0408 & 23.1 \\
\hline $\mathrm{CHCl}_{2} \mathrm{CH}_{2} \mathrm{Cl}$ & $99.9^{b}$ & 316-384 & 11 & 90.36301 & 7530.3 & 11.71063 & 9.0204 & 7.1 \\
\hline $\mathrm{CHCl}_{2} \mathrm{CH}_{2} \mathrm{CH}_{3}$ & $99.9^{b}$ & $312-362$ & 14 & 89.3533 & 6827.0 & 11.8439 & 10.8245 & 4.5 \\
\hline $\mathrm{CH}_{2} \mathrm{ClCHClCH}_{3}$ & $99.9^{b}$ & $303-368$ & 15 & 98.1061 & 7219.8 & 13.2971 & 12.6285 & 14.4 \\
\hline $\mathrm{CH}_{2} \mathrm{ClCH}_{2} \mathrm{CH}_{2} \mathrm{Cl}$ & $99.8^{b}$ & $330-393$ & 14 & 140.9922 & 9134.9 & 20.33477 & 21.1678 & 5.1 \\
\hline $\mathrm{CH}_{3} \mathrm{CCl}_{2} \mathrm{CH}_{3}$ & $99.72^{a}$ & $295-341$ & 12 & 176.6209 & 8828.3 & 27.13786 & 35.4801 & 11.6 \\
\hline $\mathrm{CH}_{3} \mathrm{CCl}_{3}$ & $99.99^{a}$ & $296-371$ & 18 & 44.7407 & 5209.8 & 4.29370 & - & 8.0 \\
\hline $\mathrm{CH}_{3} \mathrm{CCl}_{3}$ & $99.95^{b}$ & $174-223$ & 8 & 117.9294 & 4801.5 & 18.43752 & 34.8788 & 6.3 \\
\hline \multicolumn{9}{|c|}{ Alkylderivatives of adamantane } \\
\hline 1,3,5-TMA & $99.98^{a}$ & $385-482$ & 16 & 130.7579 & 10220.9 & 18.22485 & 15.8278 & 10.0 \\
\hline 1,3-DMA & $99.9^{a}$ & $352-526$ & 24 & 101.7980 & 9034.5 & 13.50183 & 10.5779 & 2.0 \\
\hline 1-EA & $99.93^{a}$ & $387-498$ & 14 & 115.7566 & 10147.8 & 15.57166 & 11.9069 & 10.6 \\
\hline \multicolumn{9}{|c|}{ Bicyclic hydrocarbons } \\
\hline cis- $\mathrm{C}_{9} \mathrm{H}_{16}$ & $99.99 a$ & $351-442$ & 18 & 118.1951 & 9158.1 & 16.27432 & 14.2522 & 9.2 \\
\hline trans- $\mathrm{C}_{9} \mathrm{H}_{16}$ & $99.98^{a}$ & $345-435$ & 18 & 107.7679 & 8639.8 & 14.60163 & 12.5083 & 11.3 \\
\hline cis- $\mathrm{C}_{10} \mathrm{H}_{18}$ & $99.87^{a}$ & $373-470$ & 19 & 129.3296 & 10107.8 & 17.94129 & 15.3346 & 7.5 \\
\hline trans- $\mathrm{C}_{10} \mathrm{H}_{18}$ & $99.98^{a}$ & $366-461$ & 19 & 105.6064 & 9031.6 & 14.11863 & 11.3055 & 5.1 \\
\hline \multicolumn{9}{|c|}{ Bicyclic perfluorocarbons } \\
\hline cis- $\mathrm{C}_{9} \mathrm{~F}_{16}$ & $99.69^{a}$ & $316-392$ & 17 & 160.6582 & 9773.9 & 22.20257 & 21.4086 & 8.0 \\
\hline trans- $\mathrm{C}_{9} \mathrm{~F}_{16}$ & $99.40^{a}$ & 314-389 & 17 & 153.5136 & 9444.2 & 21.06404 & 20.3262 & 6.0 \\
\hline cis- $\mathrm{C}_{10} \mathrm{~F}_{18}$ & $99.57^{a}$ & $315-416$ & 19 & 218.3225 & 12353.8 & 32.91383 & 34.7585 & 6.4 \\
\hline trans- $\mathrm{C}_{10} \mathrm{~F}_{18}$ & $99.46^{a}$ & $313-414$ & 18 & 195.0918 & 11539.7 & 29.04017 & 29.8875 & 10.9 \\
\hline $\mathrm{C}_{5} \mathrm{~F}_{10} \mathrm{~N}-\mathrm{C}_{6} \mathrm{~F}_{10}-\mathrm{CF}_{3}$ & $99.66^{a}$ & $374-461$ & 18 & 210.0577 & 13500.8 & 30.83011 & 28.0834 & 5.0 \\
\hline
\end{tabular}

${ }^{a}$ Adiabatic calorimetric; ${ }^{b}$ DSC.

Table 1. Thermodynamic parameters of comparative ebulliometry for compounds studied: freons; halogen -ethanes and -propanes; 1,3-dimethyladamahtane [1,3-DMA], 1,3,5trimethyladamahtane [1,3,5-TMA] and 1-ethyladamahtane [1-EA]; perfluorobicyclo $(4,3,0)$ nonanes [cis- and trans- $\mathrm{C}_{9} \mathrm{~F}_{16}$ ], bicyclo $(4,3,0)$ nonanes, [cis- and trans- $\mathrm{C}_{9} \mathrm{H}_{16}$ ], perfluorobicyclo $(4,4,0)$ decane, [cis- and trans- $\mathrm{C}_{10} \mathrm{~F}_{18}$ ], bicyclo $(4,4,0)$ decanes [cis- and trans- $\mathrm{C}_{10} \mathrm{H}_{18}$ ]; perfluoro-N-(4-methyl-cyclohexyl)piperidine $\left[\mathrm{C}_{5} \mathrm{~F}_{10} \mathrm{~N}-\mathrm{C}_{6} \mathrm{~F}_{10}-\mathrm{CF}_{3}\right]$ (Varushchenko et al., 2007; Boublik et al., 1984) 
is satisfied, the parameter $\alpha_{3}(\mathrm{D})$ may be accepted as a reliable one. Here $F$ and $F_{0.05}(1, f)$ denote evaluated and tabulated values of the $F$-criterion, and $f$ is a number of degrees of freedom. Comparing the criteria $F$ and $F_{0.05}(1, f)$ according to $(6)$ showed an adequate fit of the $p T$ parameters.

Table 1 summarizes the purity of the compounds determined by gas - liquid chromatography [g.l.c.] and adiabatic calorimetry, the temperature interval, $\Delta T(p T)$, and number, $n$, of $p T$-parameters, the coefficients of equations (4) and (5) and mean-square deviation [MSD] of calculated $p_{\text {calc }}$-values from experimental ones, $p$,

$$
S_{p}= \pm\left\{\sum\left(p-p_{\text {calc }}\right)^{2} /(n-4)\right\}^{1 / 2}
$$

\subsection{The enthalpy of vaporization}

Experimental determinations of the enthalpies of vaporization were carried out by direct calorimetric methods and by indirect ones, on the basis of the temperature dependences of saturation vapor pressures. The first method is more precise but the second one is more often used because of it's applicability for wider series of the substances.

The enthalpies of vaporization of some compounds under study were determined at $T=$ 298.15 K by calorimetric method using a carrier gas (nitrogen) (Wadsö, 1966). The method is based on measuring the energy dissipated in calorimeter for compensation of the endothermic vaporization effect. The carrier gas was employed for hastening an evaporation process and, thus, for increasing an accuracy. A modified LKB 8721-3 setup consists of some commercial parts, namely calorimetric vessel with an air brass jacket and a carrier gas system and three missing parts designed in (Varushchenko et. al., 1977): precise water thermostat, electrical scheme, and an air thermostat. The latter replaced a thermostated room that was provided for operating by this method. The calorimeter is intended for the substances with vapor pressures from $0.066 \mathrm{kPa}$ to $26.6 \mathrm{kPa}$ at $298 \mathrm{~K}$ (or normal boiling temperatures from (335 to 470$) \mathrm{K}$ ). A mass (0.5 to 1.0) $\mathrm{g}$ of substance was required for a series from 6 to 8 experiments.

The calorimetric experiment was conducted at an adiabatic and, at the same time, at isothermal conditions. The temperature of the calorimetric vessel measured by a thermistor was maintained constant and equal to that of the thermostat $(298.15 \pm 0.02) \mathrm{K}$. Electrical energy used for compensation of the energy of vaporization (20 to 40) J was measured by a potentiometer method with accuracy 0.01 per cent. The mass, $m$, of a substance evaporated ( 0.07 to 0.3$) \mathrm{g}$ was determined to $\pm 1 \cdot 10^{-4} \mathrm{~g}$ as the difference between masses of calorimetric vessel before and after an experiment. As the calorimeter was non-hermetic, the main error in mass determination arose from a loss of substance in weighing the vessel due to connecting and disconnecting it with the calorimetric system. All preliminary procedures such as filling the vessel with liquid, weighing it, and placing into its air jacket were made inside an air thermostat at $T \approx 298 \mathrm{~K}$. In so doing, we reduced a loss of the substance from the vessel and the temperature over fall of the latter.

The value of $\Delta_{\text {vap }} H$ was corrected for a small quantity of energy absorbed during the passage of nitrogen through the calorimeter under low pressure. The calorimeter was tested by measuring the enthalpies of vaporization of $n$-alkanes from $\mathrm{C}_{6}$ to $\mathrm{C}_{10}$. Obtained values of 
$\Delta_{\text {vap }} H$ at $T=298.15 \mathrm{~K}$ agree with well established literature values (Majer \& Svoboda, 1985) within (0.2 to 0.5$)$ per cent.

A main method of determination of the enthalpies of vaporization is until now an indirect one based on the temperature dependence of the vapor pressure. This is caused by a less complicated technique for precise vapor pressure determinations than direct calorimetric measurements of $\Delta_{v a p} H_{m}$. The best-accuracy estimations of $\Delta_{v a p} H_{m}$ values are attained for a moderate range of vapor pressure (5 to 150$) \mathrm{kPa}$. The literature data on the enthalpies of vaporization obtained by indirect method are usually published without uncertainties, that can be explained by fitting the $p T$-parameters with $\ln (p)=f(T)$ equations, coefficients of which were correlated. An accuracy determination of the enthalpies of vaporization in indirect method is given in Appendix. The $\Delta_{\text {vap }} H_{m}$ values obtained by indirect method were computed by equation (5) using the $\Delta Z$ difference which took into account the vapor deviation from ideality and volume changes of both phases. The $\Delta Z$ values were calculated from formula:

$$
\Delta Z=\{p /(R \cdot T)\} \cdot\left\{V_{m}(g)-V_{m}(l i q)\right\} .
$$

The molar volume $V_{m}($ liq $)$ of liquid was evaluated on the basis of density; an adequate value for the volume of vapor, $V_{m}(g)$, was calculated from the volume-explicit virial expansion truncated after the second virial coefficient $B_{v}$. The values of $B_{v}$ were evaluated on the basis of critical quantities (part 4.2) by the Tsonopolous extension of Pitzer and Curl's method (Poling et al., 2001). Comparing two series of $\Delta Z$ values estimated from experimental and calculated values of $V_{m}(g)$ of hydrocarbons enable us to accept the errors of $\Delta \mathrm{Z}$ evaluation $\leq 1$ per cent.

Freons and halogenalkanes. Table 2 presents the normal boiling temperatures, $T_{n . b .}{ }^{\prime}$ and the enthalpies of vaporization at $T=298.15 \mathrm{~K}$ and $T_{n . b}$. for freons and hologenalkanes, calculated from equation (4) and (5), respectively and calorimetric $\Delta_{v a p} H_{m}$ values.

The enthalpies of vaporization obtained both by direct and indirect methods at the saturated vapor pressure, were recalculated to the standard values by means of correction $\delta\left(\Delta H_{m}\right)=p \cdot\left\{T \cdot\left(d B_{v} / d T\right)-B_{v}\right\}$. The reliability of the calculated $\Delta_{v a p} H_{m}$ values were proved by their agreement with the calorimetric ones within the error limits (Table 2). Due to smaller extrapolation intervals, the errors of the enthalpies of vaporization at the normal boiling temperatures are less than $\Delta_{\text {vap }} H_{m}(298.15 K)$ values. Extrapolation capabilities of equations (3) and (5) were verified by comparison of calculated $\Delta_{\text {vap }} H_{m}$ values at $T=298.15 \mathrm{~K}$ with experimental ones for some well studied alkanes and alkanethiols (Boublik et al., 1984). It has been shown that these equations allowed us to estimate the $\Delta_{\text {vap }} H_{m}$ values with uncertainties $\leq 2$ per cent in extrapolation intervals $\Delta T \leq 50 \mathrm{~K}$.

Mutual congruence of some thermodynamic properties in set of related compounds (Table 2) can be drawn from comparison of these properties in dependence on some physicochemical characteristics having influence upon intermolecular interactions in liquid state.

Fig. 3 represent critical temperatures, $T_{\mathcal{C}}$, normal boiling temperatures, $T_{n . b}{ }^{\prime}$ and enthalpies of vaporization, $\Delta_{v a p} H_{m}(298.15 K)$, for freons and chloroalkanes $C_{2}, C_{3}$ depending on the dipole moments, $\mu_{(\text {liq })}$, and coefficients of molecular packing, $K_{m}$, in the liquids. The 


\begin{tabular}{|c|c|c|c|c|c|}
\hline \multicolumn{2}{|c|}{ Compounds } & $\begin{array}{c}T_{n . b .} \\
\mathrm{K}\end{array}$ & $\begin{array}{c}\Delta_{\text {vap }} H_{m}^{0}(298.15 K) \\
(\text { calor }) \\
\mathrm{kJ} \cdot \mathrm{mol}^{-1}\end{array}$ & $\begin{array}{c}\Delta_{\text {vap }} H_{m}^{0}(298.15 K) \\
(p-T) \\
\mathrm{kJ} \cdot \mathrm{mol}^{-1}\end{array}$ & $\begin{array}{c}\Delta_{\text {vap }} H_{m}^{0}\left(T_{n . b .}\right) \\
(p-T) \\
\mathrm{kJ} \cdot \mathrm{mol}^{-1}\end{array}$ \\
\hline \multicolumn{6}{|c|}{ Freons and halogen -ethanes and -propanes } \\
\hline $\mathrm{CFCl}_{2} \mathrm{CFCl}_{2}$ & $\mathrm{R}-112$ & $366.00 \pm 0.01$ & - & $34.98 \pm 0.39$ & $31.26 \pm 0.33$ \\
\hline $\mathrm{CF}_{2} \mathrm{ClCFCl}_{2}$ & R-113 & $320.76 \pm 0.01$ & $28.61 \pm 0.09$ & $28.71 \pm 0.41$ & $27.21 \pm 0.44$ \\
\hline $\mathrm{CF}_{2} \mathrm{ClCF}_{2} \mathrm{Cl}$ & R-114 & 276.65 & - & - & $24.9 \pm \pm 1.1$ \\
\hline $\mathrm{CF}_{2} \mathrm{BrCF}_{2} \mathrm{Br}$ & $\mathrm{R}-114 \mathrm{~b} 2$ & $320.36 \pm 0.01$ & $28.61 \pm 0.09$ & $28.63 \pm 0.34$ & $27.11 \pm 0.32$ \\
\hline $\mathrm{CF}_{2} \mathrm{ClCHCl}_{2}$ & $\mathrm{R}-122$ & $345.01 \pm 0.01$ & $32.91 \pm 0.09$ & $32.84 \pm 0.40$ & $29.84 \pm 0.36$ \\
\hline $\mathrm{CFCl}_{2} \mathrm{CHFCl}$ & $\mathrm{R}-122 \mathrm{a}$ & $346.34 \pm 0.01$ & $33.10 \pm 0.06$ & $33.04 \pm 0.36$ & $29.95 \pm 0.35$ \\
\hline $\mathrm{CF}_{3} \mathrm{CHCl}_{2}$ & $\mathrm{R}-123$ & $300.981 \pm 0.008$ & - & $26.35 \pm 0.33$ & $26.14 \pm 0.34$ \\
\hline $\mathrm{CF}_{2} \mathrm{ClCHFCl}$ & $\mathrm{R}-123 \mathrm{a}$ & $303.02 \pm 0.01$ & - & $26.82 \pm 0.28$ & $26.45 \pm 0.32$ \\
\hline $\mathrm{CF}_{3} \mathrm{CHClBr}$ & R-123b1 & $323.41 \pm 0.01$ & $29.80 \pm 0.09$ & $30.01 \pm 0.33$ & $28.36 \pm 0.31$ \\
\hline $\mathrm{CF}_{3} \mathrm{CH}_{2} \mathrm{CH}_{2} \mathrm{Cl}$ & R-253fa & $318.84 \pm 0.01$ & - & $29.86 \pm 0.39$ & $28.39 \pm 0.40$ \\
\hline $\mathrm{CF}_{3} \mathrm{CH}_{2} \mathrm{CHCl}_{2}$ & $\mathrm{R}-243$ & $345.47 \pm 0.01$ & $34.05 \pm 0.04$ & $34.40 \pm 0.57$ & $31.14 \pm 0.47$ \\
\hline $\mathrm{CF}_{3} \mathrm{CH}_{2} \mathrm{CCl}_{3}$ & $\mathrm{R}-233$ & $368.28 \pm 0.01$ & $36.76 \pm 0.08$ & $37.4 \pm 1.4$ & $32.62 \pm 0.57$ \\
\hline $\mathrm{CF}_{3} \mathrm{CH}_{2} \mathrm{CFCl}_{2}$ & $\mathrm{R}-234 \mathrm{fb}$ & $333.74 \pm 0.01$ & - & $33.59 \pm 0.65$ & $29.80 \pm 0.62$ \\
\hline $\mathrm{CHCl}_{2} \mathrm{CH}_{3}$ & 1,1-DClE & $330.35 \pm 0.01$ & $30.62 \pm 0.14$ & $31.12 \pm 0.34$ & $29.24 \pm 0.35$ \\
\hline $\mathrm{CH}_{2} \mathrm{ClCH}_{2} \mathrm{Cl}$ & 1,2-DClE & $356.61 \pm 0.01$ & $32.15 \pm 0.01$ & $35.36 \pm 0.39$ & $32.18 \pm 0.35$ \\
\hline $\mathrm{CH}_{2} \mathrm{BrCH}_{2} \mathrm{Br}$ & 1,2-DBrE & $404.55 \pm 0.01$ & $41.73 \pm 0.02$ & $41.97 \pm 0.95$ & $36.23 \pm 0.51$ \\
\hline $\mathrm{CHCl}_{2} \mathrm{CH}_{2} \mathrm{Cl}$ & 1,1,2-TClE & $386.98 \pm 0.01$ & $40.28 \pm 0.10$ & $40.23 \pm 0.56$ & $35.09 \pm 0.42$ \\
\hline $\mathrm{CH}_{3} \mathrm{CCl}_{3}$ & 1,1,1-TClE & $347.21 \pm 0.01$ & $32.62 \pm 0.09$ & $32.58 \pm 0.35$ & $29.89 \pm 0.34$ \\
\hline $\mathrm{CHCl}_{2} \mathrm{CH}_{2} \mathrm{CH}_{3}$ & 1,1-DClP & $361.53 \pm 0.01$ & $35.10 \pm 0.11$ & $35.34 \pm 0.45$ & $31.85 \pm 0.35$ \\
\hline $\mathrm{CH}_{2} \mathrm{ClCHClCH}_{3}$ & 1,2-DClP & $369.50 \pm 0.01$ & $36.20 \pm 0.08$ & $36.37 \pm 0.49$ & $32.45 \pm 0.41$ \\
\hline $\mathrm{CH}_{2} \mathrm{ClCH}_{2} \mathrm{CH}_{2} \mathrm{Cl}$ & 1,3-DClP & $393.95 \pm 0.01$ & $40.75 \pm 0.04$ & $41.18 \pm 0.55$ & $35.56 \pm 0.38$ \\
\hline $\mathrm{CH}_{3} \mathrm{CCl}_{2} \mathrm{CH}_{3}$ & 2,2-DClP & $342.67 \pm 0.01$ & - & $32.22 \pm 0.38$ & $29.66 \pm 0.37$ \\
\hline $\mathrm{CH}_{3} \mathrm{CFl}_{3}$ & R-143a & $225.85 \pm 0.01$ & - & - & $19.40 \pm 0.24$ \\
\hline
\end{tabular}

Table 2. Normal boiling temperatures, $T_{n . b},{ }^{\prime}$ molar enthalpies of vaporization, $\Delta_{\text {vap }} H_{m}(298.15 \mathrm{~K})$, measured calorimetrically and calculated from $p T$ data at $T=298.15 \mathrm{~K}$ and $T_{n . b}$ for some freons and halogen-alkanes (Varushchenko et al., 2007; Majer \& Svoboda, 1985; Boublik et al., 1984) 

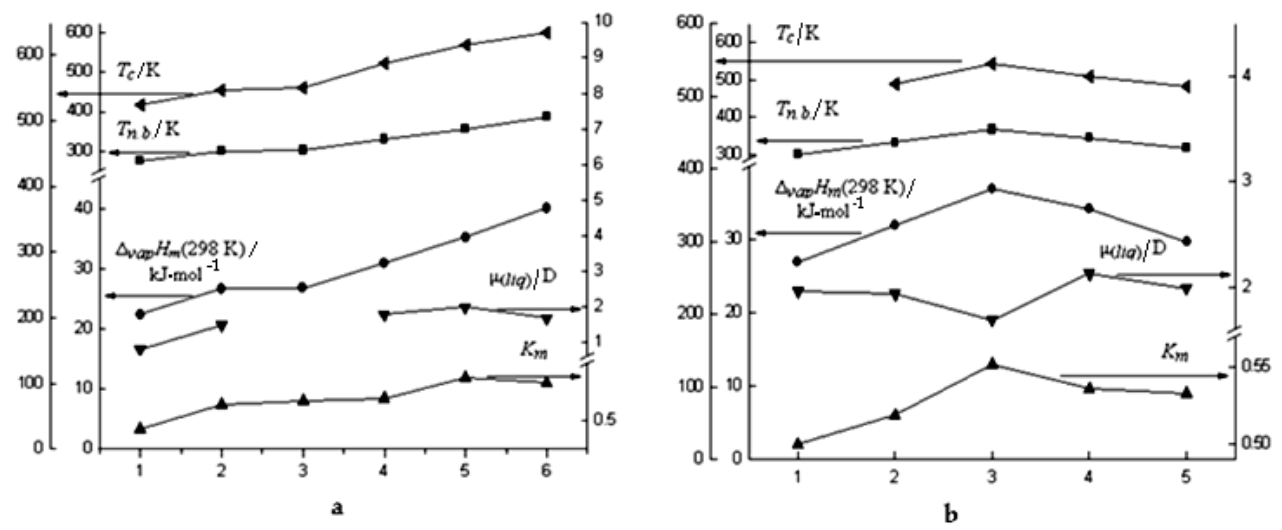

Fig. 3. Variations of thermodynamic properties $T_{\mathcal{C}}, T_{n . b .}$ and $\Delta_{\text {vap }} H_{m}(298.15 K)$ in dependence on the dipole moments, $\mu_{(\text {liq })}$, and coefficients of molecular packing, $K_{m}$, in series of liquid halogenated ethanes (a): $\mathrm{CF}_{3} \mathrm{CHCl}_{2}$ [1], $\mathrm{CF}_{2} \mathrm{ClCHFCl}$ [2], $\mathrm{CF}_{2} \mathrm{ClCFCl}_{2}$ [3], $\mathrm{CH}_{3} \mathrm{CHCl}_{2}$ [4], $\mathrm{CH}_{2} \mathrm{ClCH}_{2} \mathrm{Cl}$ [5], $\mathrm{CHCl}_{2} \mathrm{CH}_{2} \mathrm{Cl}$ [6]; and propanes (b): $\mathrm{CF}_{3} \mathrm{CH}_{2} \mathrm{CF}_{2} \mathrm{Cl}$ [1], $\mathrm{CF}_{3} \mathrm{CH}_{2} \mathrm{CFCl}_{2}$ [2], $\mathrm{CF}_{3} \mathrm{CH}_{2} \mathrm{CCl}_{3}$ [3], $\mathrm{CF}_{3} \mathrm{CH}_{2} \mathrm{CHCl}_{2}$ [4], $\mathrm{CF}_{3} \mathrm{CH}_{2} \mathrm{CH}_{2} \mathrm{Cl}$ [5]

coefficients $K_{m}$ were calculated by analogy with (Varushchenko et al., 2007). In spite of the large atomic weight of fluorine in comparison with hydrogen, thermodynamic values of compounds decrease when hydrogen is substituted for fluorine that can be explained by decreasing of the $\mu_{(\text {liq })}$ and $K_{m}$ parameters. Minimum $T_{\mathcal{C}}, T_{n . b .}$, and $\Delta_{v a p} H_{m}(298.15 K)$ values are inherent to completely halogenated 1,1,1-trifluoro-2,2-dichloroethane, which has the lowest values of dipole moment and $K_{m}$ coefficient. Maximum values of corresponding properties are observed for the most polar compounds, 1,1,2,-trichloroetane, the gauche conformer of which is stabilized by the dipole interaction in the liquid phase.

Analysis of the data shown in Fig. 3 allows to conclude that the values of critical and normal boiling temperatures and enthalpy of vaporization vary in a series of compounds according to the combined action of the parameters responsible for intermolecular interactions and short range order of the liquid phase, thus proving the mutual consistency of the thermodynamic data in the series of halogenated ethane and propane.

Cyclic perfluorocarbons and hydrocarbons. A thermodynamic study of perfluorated cyclic organic compounds has scientific and practical importance. Perfluorocarbons [PFC] have high chemical and thermal stability, absolute biological inertness, and weak intermolecular interactions [IMI]. The combination of these properties can be assigned to high C-F bond strength and the shielding effect of fluorine atoms towards the carbon framework. The weakness of IMI is responsible for the ability of PFC to dissolve and transfer considerable amounts of gases, in particular, oxygen and carbon dioxide. On account of these properties, PFC have found wide application in biology and medicine as efficient gas-transfer media (blood substitutes). 


\begin{tabular}{|c|c|c|c|c|c|}
\hline Compounds & $\begin{array}{c}T_{n . b .} \\
\mathrm{K}\end{array}$ & $\begin{array}{c}\Delta_{\text {vap }} H_{m}^{0}(298.15 K) \\
(\text { calor }) \\
\mathrm{kJ} \cdot \mathrm{mol}^{-1}\end{array}$ & \begin{tabular}{|c|}
$\Delta_{\text {vap }} H_{m}^{0}(298.15 K)$ \\
$(p-T)$, \\
$\mathrm{kJ} \cdot \mathrm{mol}^{-1}$ \\
\end{tabular} & $\begin{array}{c}\Delta_{\text {vap }} H_{m}^{0}\left(T_{n . b .}\right) \\
(p-T) \\
\mathrm{kJ} \cdot \mathrm{mol}^{-1}\end{array}$ & $\begin{array}{c}\varphi\left(\mathrm{O}_{2}\right) \\
(298.15), \\
\mathrm{cm}^{3} / 100 \mathrm{ml}\end{array}$ \\
\hline \multicolumn{6}{|c|}{ Bicyclic perfluorocarbons } \\
\hline cis- $\mathrm{C}_{9} \mathrm{~F}_{16}$ & $391.52 \pm 0.01$ & $41.98 \pm 0.14$ & $41.95 \pm 0.52$ & $34.43 \pm 0.37$ & 44.91 \\
\hline trans- $\mathrm{C}_{9} \mathrm{~F}_{16}$ & $389.02 \pm 0.01$ & $41.34 \pm 0.05$ & $41.22 \pm 0.51$ & $34.11 \pm 0.36$ & 46.11 \\
\hline cis- $\mathrm{C}_{10} \mathrm{~F}_{18}$ & $416.96 \pm 0.01$ & $46.19 \pm 0.12$ & $46.79 \pm 0.62$ & $36.57 \pm 0.43$ & 40.30 \\
\hline trans $-\mathrm{C}_{10} \mathrm{~F}_{18}$ & $414.70 \pm 0.01$ & $45.40 \pm 0.08$ & $46.02 \pm 0.60$ & $36.26 \pm 0.43$ & 41.10 \\
\hline $\begin{array}{c}\mathrm{C}_{5} \mathrm{~F}_{10} \mathrm{~N}-\mathrm{C}_{6} \mathrm{~F}_{10-} \\
\mathrm{CF}_{3} \\
\end{array}$ & $460.74 \pm 0.01$ & $56.56 \pm 0.24$ & $56.58 \pm 0.88$ & $40.68 \pm 0.44$ & 34.30 \\
\hline \multicolumn{6}{|c|}{ Bicyclic hydrocarbons } \\
\hline cis- $\mathrm{C}_{9} \mathrm{H}_{16}{ }^{a}$ & $440.99 \pm 0.01$ & - & \begin{tabular}{|l|l|}
$46.34 \pm 0.82$ \\
\end{tabular} & $38.01 \pm 0.44$ & 25.22 \\
\hline trans- $\mathrm{C}_{9} \mathrm{H}_{16}{ }^{a}$ & $434.22 \pm 0.01$ & - & $44.88 \pm 0.78$ & $37.21 \pm 0.43$ & 28.84 \\
\hline cis- $\mathrm{C}_{10} \mathrm{H}_{18}{ }^{a}$ & $468.93 \pm 0.01$ & - & $50.90 \pm 0.94$ & $40.46 \pm 0.45$ & 21.25 \\
\hline trans $-\mathrm{C}_{10} \mathrm{H}_{18}{ }^{a}$ & $460.43 \pm 0.01$ & \begin{tabular}{|c|}
- \\
\end{tabular} & $48.45 \pm 0.78$ & $39.29 \pm 0.43$ & 25.05 \\
\hline \multicolumn{6}{|c|}{ Alkylderivatives of adamantane } \\
\hline 1,3,5-TMA & $483.31 \pm 0.01$ & \begin{tabular}{|l|}
$51.74 \pm 0.20$ \\
\end{tabular} & $51.50 \pm 0.52$ & $40.52 \pm 0.50$ & - \\
\hline 1,3-DMA ${ }^{a}$ & 476.441 & $49.71 \pm 0.20$ & $49.47 \pm 0.54$ & $39.71 \pm 0.41$ & - \\
\hline 1-EA & $498.86 \pm 0.01$ & $54.96 \pm 0.28$ & $54.6 \pm 1.3$ & $42.56 \pm 0.51$ & - \\
\hline
\end{tabular}

${ }_{a} T_{n . b .}$ and $\Delta_{\text {vap }} H_{m}(298.15 K)$ values were calculated from literature data of (Boublik et. al., 1984).

Table 3. Normal boiling temperatures, $T_{n . b .}$, molar enthalpies of vaporization, $\Delta_{\text {vap }} H_{m}(298.15 K)$, obtained by direct and indirect methods, and oxygen capacities, $\varphi\left(\mathrm{O}_{2}\right)(298.15 \mathrm{~K})$, for bicyclic hydrocarbons, perfluorocarbons, and derivatives of adamantine

The saturated vapor pressure of bicyclic PFC at temperature $(310 \mathrm{~K})$ of the human body, $p_{S}^{310}$, is one of the key properties of the blood substitute, which ranges from 0.16 to $2.66 \mathrm{kPa}$. A stability of an aqueous emulsion of fluorocarbon and its delivery rate from the body depends on $p_{S}^{310}$ value. Medicine employs bicyclic perfluorocarbon composition with high and low vapor pressures. Perfluoro-N-(4-methylcyclohexyl)piperidine, $\mathrm{C}_{5} \mathrm{~F}_{10} \mathrm{~N}-\mathrm{C}_{6} \mathrm{~F}_{10^{-}}$ $\mathrm{CF}_{3}$, having the low value of $p_{S}^{310}=0.157 \mathrm{kPa}$, is a component of "Ftorosan" (Russia) blood substitute in mixture with cis- and trans- perfluorodecalines, which have higher (1.54 and 1.72) $\mathrm{kPa}$ values of $p_{S}^{310}$, respectively. Another key property of the blood substitutes is an oxygen capacity, $\varphi\left(\mathrm{O}_{2}\right)\left(\mathrm{cm}^{3} / 100 \mathrm{ml}\right)$, which is defined as a volume of oxygen, dissolved in $100 \mathrm{ml}$ of the liquid. The $\varphi\left(\mathrm{O}_{2}\right)$ values were evaluated on the basis of the enthalpies of vaporization by empirical method developed within a theory of regular solutions (Lawson et al., 1978).

Table 3 presents derived thermodynamic values of cyclic compounds. The values of the normal boiling temperatures and the enthalpies of vaporization of cis-isomers are more than 
those of trans-isomers in the series of perfluorobicyclo-nonanes and -decanes and their hydrocarbons analogues. Despite the more molecular mass, the normal boiling temperatures $T_{n . b .}$ and the $\Delta_{\text {vap }} H_{m}$ values of the perfluorocarbons are less than those of the hydrocarbons. On the contrary, the oxygen capacities are two times more in the series of perfluorocarbons which can be explanted by more poor intermolecular interactions of PFC. Fig. 4 presents the critical temperatures, enthalpies of vaporization, and oxygen capacities, $\varphi\left(\mathrm{O}_{2}\right)$, for cis- and trans- perfluorobicyclo(4,3,0)nonanes (1 and 2), for components of Ftorosan blood substitute (Ries, 1991), namely perfluorobicyclo(4,4,0)-decanes (3 and 4), perfluoro-N-(4-methylcyclohexyl)piperidine (5), and for some of their hydrocarbon analogues (6-9), respectively.

Due to smaller energies of intermolecular interactions, the critical temperatures and enthalpies of vaporization of perfluorocarbons are less, but oxygen capacities are more, than appropriate properties of appropriate hydrocarbons.

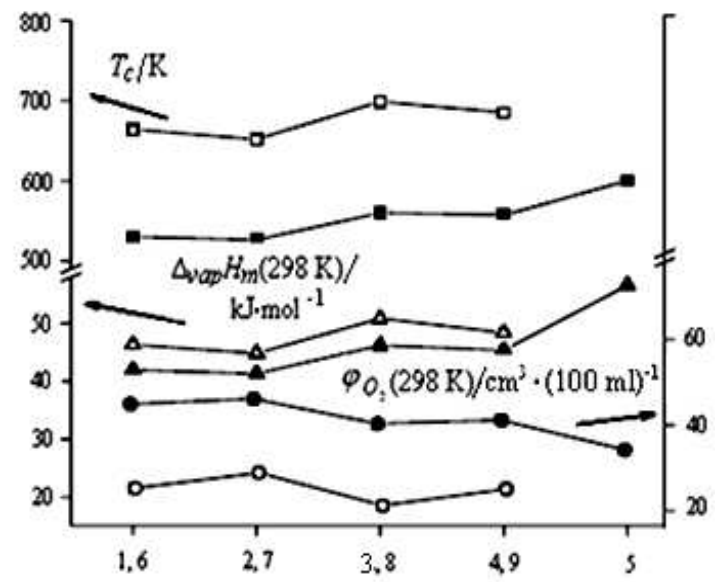

Fig. 4. Critical temperatures, $T_{\mathcal{C}}$, enthalpies of vaporization, $\Delta_{v a p} H_{m}(298.15 \mathrm{~K})$, and oxygen capacities, $\varphi\left(\mathrm{O}_{2}\right)(298.15 \mathrm{~K})$, for perfluorinated compounds $1-5(\boldsymbol{\Delta} \bullet)$ and their hydrocarbon analogues 6-9 $(\square \Delta \circ)$

Despite the more molecular mass, the normal boiling temperatures and enthalpies of vaporization of perfluorocarbons are less than those of appropriate hydrocarbon. This can be explained less coefficients of molecular packing, $K_{m}$, and therefore by more intermolecular distances, and as a consequence less intermolecular interactions of perfluorocarbons in comparison with their hydrogen - containing counterparts.

\subsection{The vapor pressure and enthalpies of vaporization of the hard-volatile compounds}

The saturation vapor pressures of the solid and liquid substances having $p<1 \mathrm{kPa}$ were determined by a dynamic method of evaporating the sample in a stream of the carrier inert gas. In calculation of the enthalpy of vaporization, the volume of vapor is well described by the ideal gas law and the volume of liquid can be easily neglected without introducing 
essential error into the $\Delta_{v a p} H_{m}$ value. But the $d P / d T$ or $d \ln (p) / d T$ derivatives are determined not enough reliably because the saturation vapor pressure is a weak function of the temperature. Thus, an accuracy of determination of the enthalpy of vaporization is restricted for the compounds with low vapor pressures at about $298 \mathrm{~K}$ temperature.

The temperature dependences of the vapor pressures for the ferrocene derivatives [FD] were determined by a transpiration method elaborated and fully described by Verevkin S.P. and coathers (Emel'yanenko et al., 2007). Here, only the main features of the method are given. The determination of the vapor pressure is based on the measurements of the mass of substance transpired in the stream of carrier gas (nitrogen) and the volume of the gas flowing. The vapor pressure of the substances was obtained by Dalton law for partial vapor pressures of the ideal gas mixture. A sample of the substance $(\sim 0.5 \mathrm{~g})$ was placed into the Utube, temperature of which was controlled with accuracy $\pm 0.1 \mathrm{~K}$. A nitrogen flow, controlled by a precision Hoke valve and measured with a bubble gauge, was passed through the tube. The transferred substance was condensed in a cooled trap and was analyzed chromatographically using the external standard (hydrocarbons). The rate of the nitrogen flow was adjusted to ensure that the condensed and vapor phases were in stable equilibrium. The saturation vapor pressure $p_{\text {sat }}$ was calculated by the formula:

$$
p_{\text {sat }}=m R T / V M
$$

where $V=V\left(N_{2}\right)+\mathrm{V}(\mathrm{DF}) ; R=8.314472 ; m$ and $M$ are the mass of the sample under study and molecular weight of FD, respectively; $V\left(N_{2}\right)$ and $V(F D)$ are the nitrogen and FD volumes, respectively, $V\left(N_{2}\right)>\mathrm{V}(\mathrm{FD})$; and $T$ is the $\mathrm{U}$-tube temperature. The $V\left(N_{2}\right)$ value was determined from the flow rate and the measurement time.

The $p T$ parameters of the solid FD were measured in the pressure and temperature intervals from (0.01/0.11 to $0.44 / 4.9) \mathrm{Pa}$ and from (311/342 to $341 / 379) \mathrm{K}$, respectively. Appropriate pressure and temperature intervals for the liquid FD were from $(0.3 / 1.87$ to $7.88 / 130) \mathrm{Pa}$ and from $(298 / 384$ to $358 / 430) \mathrm{K}$, respectively. The vapor pressures of FD were approximated by equation:

$$
R \cdot \ln (p)=a+b / T+\Delta C_{p, m} \cdot \ln \left(T / T_{s t}\right)
$$

where $a$ and $b$ are coefficients, $\Delta C_{p, m}=C_{p, m}(g)-C_{p, m}(\mathrm{cr} / \mathrm{liq})$ is the difference between the heat capacities of the vapor and condensed phases, and $T_{s t}=298.15 \mathrm{~K}$ is the standard temperature (arbitrarily chosen). Equation (9) was deduced by integration of the correlation $-R \cdot[(d \ln (p)) / d(1 / T)]=\Delta_{v a p} H_{m, T}+\Delta C_{p, m}\left(T-T_{s t}\right)$ (Kulikov et al., 2001). The latter was obtained on the basis of Clausius-Clapeyron equation $R \cdot[(d \ln (p)) / d(1 / T)]=\Delta_{\text {vap }} H_{m}$ in approximation of $\Delta_{\text {liq }}^{g} V_{m} \approx R \cdot T / p$ and derivative $d\left(\Delta_{v a p} H_{m}\right) / d T=\Delta C_{p, m}$ by assuming that $\Delta_{\text {liq }}^{g} C_{p, m}$ value is independent on the temperature in the $p T$ interval under study. The enthalpy of vaporization was calculated by the formula:

$$
\Delta_{\text {vap }} H_{m}\left(\Delta_{\text {sub }} H_{m}\right)=-b+\Delta C_{p, m} \cdot T
$$

obtained by differentiation of equation (9) with respect to $1 / T$. The ideal gas heat capacities of the ferrocene derivatives [FD] were obtained by additive Chickos and Acree method (Chikos \& Acree Jr., 2003) that is defined as "an atom together with all of its ligands". 
Table 4 lists the purity of ferrocene derivatives determined by adiabatic calorimetry (part 3.2), coefficients $a$ and $b$ of equations (9) and (10), and enthalpies and entropies of vaporization and sublimation of $\mathrm{FD}$ at $T=298.15 \mathrm{~K}$.

\begin{tabular}{|c|c|c|c|c|c|c|c|}
\hline \multirow{2}{*}{$\begin{array}{l}\text { Comp } \\
\text { ounds }\end{array}$} & \multirow{2}{*}{$\begin{array}{l}\text { Purity, } \\
\text { mol. \% }\end{array}$} & \multirow[t]{2}{*}{$a$} & \multirow[t]{2}{*}{$-b$} & $\Delta_{v a p} H_{m}^{O}(T)$ & $\Delta_{s u b} H_{m}^{O}(T)$ & $\Delta_{\text {vap }} S_{m}^{O}(T)$ & $\Delta_{s u b} S_{m}^{O}(T)$ \\
\hline & & & & \multicolumn{2}{|c|}{$\mathrm{k} \cdot \mathrm{mol}^{-1}$} & \multicolumn{2}{|c|}{$\mathrm{J} \cdot \mathrm{K}^{-1} \cdot \mathrm{mol}^{-1}$} \\
\hline \multirow[t]{2}{*}{ FM } & \multirow{2}{*}{$\begin{array}{c}97.56^{a} ; \\
99.0^{b}\end{array}$} & 339.7 & 111826.0 & & $102.8 \pm 2.0$ & & $344.8 \pm 6.4$ \\
\hline & & 359.5 & 115237.0 & $86.97 \pm 1.7$ & & $291.7 \pm 5.7$ & \\
\hline \multirow[t]{2}{*}{$\mathrm{BF}$} & \multirow{2}{*}{$\begin{array}{c}99.47^{a} ; \\
99.7^{b}\end{array}$} & 359.9 & 121314.1 & & $109.3 \pm 2.0$ & & $366.6 \pm 6.0$ \\
\hline & & 373.9 & 124422.2 & $90.64 \pm 1.8$ & & $304.0 \pm 5.8$ & \\
\hline \multirow[t]{2}{*}{$\mathrm{BOF}$} & \multirow[t]{2}{*}{$99.6^{b}$} & 364.8 & 133682.1 & & $119.9 \pm 2.4$ & & $402.1 \pm 6.3$ \\
\hline & & 382.9 & 133533.0 & $98.2+2.0$ & & $329.4 \pm 5.2$ & \\
\hline POF & $\begin{array}{l}99.24^{a} ; \\
99.0^{b}\end{array}$ & 353.7 & 112812.1 & $80.8 \pm 1.6$ & $99.1 \pm 2.8^{c}$ & $271.0 \pm 5.0$ & $332.4 \pm 6.0$ \\
\hline$n-P F$ & $\begin{array}{c}98.93^{a} ; \\
99.0^{b}\end{array}$ & 320.5 & 95312.0 & $69.2 \pm 1.4$ & & $232.1 \pm 4.7$ & \\
\hline$i-B F$ & $\begin{array}{c}99.41^{a} ; \\
99.0^{b}\end{array}$ & 326.6 & 98138.8 & $70.7 \pm 1.5$ & & $237.1 \pm 4.9$ & \\
\hline
\end{tabular}

a Adiabatic calorimetry; $b$ DSC; $c$ the value calculated on the basis of correlation $\Delta_{\text {sub }} H_{m}=\Delta_{\text {vap }} H_{m}+\Delta_{\text {fus }} H_{m}$.

Table 4. The purity, coefficients of equations (9) and (10), enthalpies and entropies of vaporization, $\Delta_{v a p} H_{m}^{O}(T)$ and $\Delta_{v a p} S_{m}^{O}(T)$, and sublimation, $\Delta_{s u b} H_{m}^{O}(T)$ and $\Delta_{s u b} S_{m}^{O}(T)$ of ferrocenylmethanol [FM], benzylferrocene [BF], benzoylferrocene [BOF], propionylferrocene [POF], npropylferrocene $[n-P F]$, iso-butylferrocene $[i-B F]$ at $T=298.15 \mathrm{~K}$

For testing the uncertainties of transpiration method in applying to FD, a compilation of the literature data on the enthalpy of sublimation of ferrocene were carried in reference (Emel'yanenko et al., 2007). A series of $21 \Delta_{s u b} H_{m}^{O}(298.15 K)$ values ranged from $(70.3 \pm 1.0$ to $76.78 \pm 0.85) \mathrm{kJ} \cdot \mathrm{mol}^{-1}$ was obtained, the most part of the data being focused in the range between (72 and 74) $\mathrm{kJ} \cdot \mathrm{mol}^{-1}$. Uncertainties of these quantities were probably the random errors. Taking into account the uncertainties of the initial vapor pressure data making up from (1.5 to 2 ) per cent, a total value of the random and systematic errors could be $\geq 2 \%$. Therefore, the errors of the enthalpies of vaporization and sublimation as derivative values of the vapor pressure in the transpiration method were evaluated as $\pm \geq 2 \%$.

\section{The heat capacity and thermodynamic properties of the phase transitions}

A heat capacity is a capability of the substance for absorbing some quantity of the energy that increases its temperature by 1 degree $\mathrm{K}$. A measurement of the heat capacity is performed by adiabatic and isothermal methods. The first one allows attaining the most complete thermodynamic equilibrium or, in any case, the thermal balance in the calorimetric system. The adiabatic method is used for exploring the thermal processes with different times of relaxation and the metastable phases which can exist in wide temperature ranges. The heat capacities and thermodynamic properties of the phase transitions were 
investigated in this work by low-temperature adiabatic calorimetry (Varushchenko et al., 1997a).

\subsection{Experimental}

The measurements of the heat capacities were conducted in a fully automated setup, consisted of a vacuum adiabatic calorimeter, a data acquisition and control system, AK-9.02, and a personal computer, PC (Fig. 5). The setup was produced in the National Scientific and

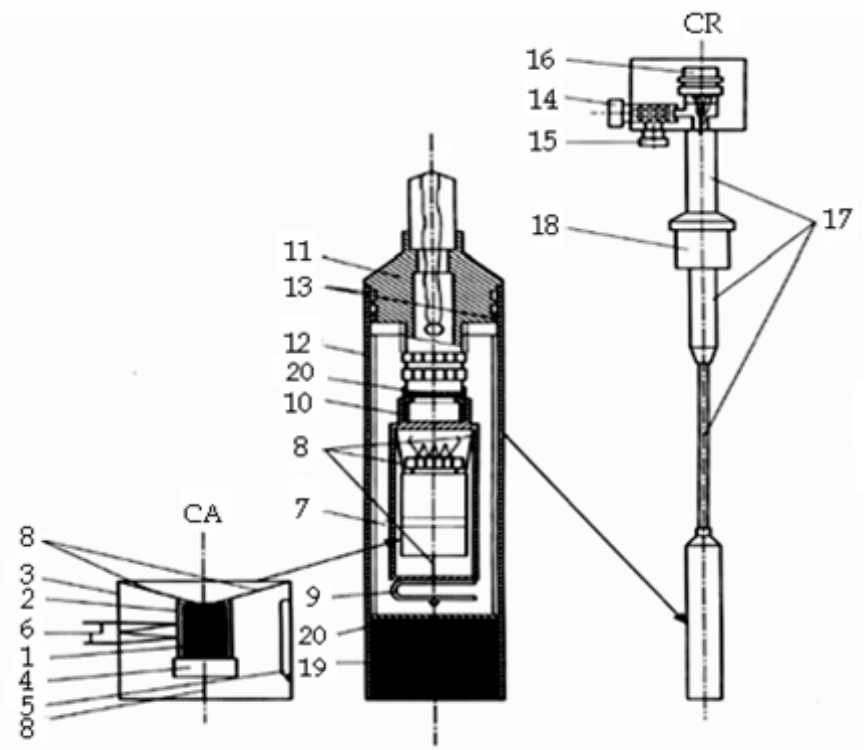

Fig. 5. The vacuum adiabatic calorimeter (CA) and cryostat (CR): (1) titanium container ( $V \sim 1$ $\left.\mathrm{cm}^{3}\right)$; (2) copper sleeve with the heater of calorimeter; (3) adiabatic shield; (4) bronze lid of the container; (5) the rhodium-iron resistance thermometer; (6) four-junction battery of $\mathrm{Cu} / \mathrm{Fe}$ - Chromel thermocouples; (7) radiation screen - aluminium- coated Dacron-like film; (8) nylon threads; (9) spring; (10) Teflon tube; (11) plug; (12) vacuum jacket; (13) grooves of the plug 11; (14) valve; (15) and (16) detachable vacuum and cable joists, respectively; (17) steel tubes; (18) coupling nut; (19) charcoal getter; (20) radiation screens.

Research Institute of Physical Technical and Radio-Technical Measurements (Mendeleevo, Moscow Region). The main principles of its construction were published in (Pavese \& Malyshev, 1994).

The calorimetric cell consists of a container, 1, a copper sleeve, 2, in which the container is tightly held, and an adiabatic shield, 3. A bronze brass lid, 4, serves for vacuum-tight sealing the container by means of indium gasket and a simple manifold. To decrease the heat capacity of the empty calorimeter, the miniature rhodium-iron resistance thermometer, 5, $\left(R_{0}=50 \Omega\right)$ was mounted on the inner surface of the adiabatic shield. The thermometer, which was calibrated on ITS-90, is destined for temperature measurements from (0.5 to 373) $\mathrm{K}$ with accuracy $\pm 3 \cdot 10^{-3} \mathrm{~K}$. The temperature difference between the calorimeter and the 
adiabatic shield is measured by a four-junction thermocouple, $6,(\mathrm{Cu}+0.1$ per cent Fe alloy against Chromel), one end of which was mounted on the copper sleeve 2 and the other one was placed on the inner surface of the adiabatic shield, 3. A manganin calorimeter heater $(R$ $=300 \Omega$ ) was wound non-inductively on the sleeve, 2 . A well-known three-lead circuit diagram was employed for wiring the current and potential leads of the heater. Since the resistances of the current leads are equal, this diagram enables us to account for the heat generated in the leads between the calorimeter and the shield. To reduce the level of heat radiation, the shield was wrapped with several layers of aluminium-coated Lavsan film, 7, (ACLF, an analog of Mylar). The container sleeve, 2, is suspended inside the adiabatic shield on three nylon threads, 8 , which are stretched by a spring, 9 (Fig. 5). The calorimeter cell has been fixed on an epoxy/fibre-glass tube, 10, of the cryostat, CR. The tube, 10, is fastened to a copper plug, 11, by means of a bayonet joint. The only removable part of the calorimeter cell is the container for the specimen.

The vacuum jacket, 12, is made from oxygen-free copper. The vacuum seal of the cryostat is provided by a KPT-8 silicon/boron nitride paste, which has high thermal conductivity value and gives a stable vacuum junction after freezing. The paste is put between the upper part of the jacket, 12, and the plug, 11, in its grooves, 13.

The top part of the cryostat (CR) has a valve, 14, detachable vacuum, 15, and cable, 16, joints; the latter connects the electrical leads of the calorimeter cell to AK-9.02 and PC. Both parts of the cryostat are jointed by the stainless steel tubes, 17 . Due to small size $(l=120 \mathrm{~mm}, d=22.5$ $\mathrm{mm})$, the cryostat is immersed directly into a commercial transportation Dewar vessels. This allows us to exclude an intermediate Dewar vessel and, thus, to reserve the coolants. A coupling nut, 18, with a Teflon shell and a rubber ring is used to fasten the cryostat airtight inside the neck of the Dewar vessel. A T-connection, fitted on the neck of the nitrogen Dewar vessel, enables us to pump out nitrogen vapors to lower the bath temperature if necessary.

The calorimeter cell is cooled down by thermal conductivity via electrical leads and by radiation heat transfer. The leads of the thermometer, heaters, and differential thermocouple form a heat shunt with the preset thermal resistance and they provide cooling of the calorimeter from room temperature to approximately $\mathrm{T}=78 \mathrm{~K}$, and from $\mathrm{T}=78 \mathrm{~K}$ down to $\mathrm{T}$ $=5 \mathrm{~K}$ for about $7 \mathrm{~h}$ in each Dewar vessel. The helium heat-exchange gas is not used for this purpose in order to avoid problems, connected with it desorption. To reduce the heat losses by radiation, the additional radiation screens, 20, are used (Fig. 5).

The data acquisition system AK-9.02 is a single unit, connected with a personal computer [PC]. The system AK-9.02 and the PC perform the measurements of all values that are necessary for the determination of the heat capacity, as well as the control of the measurement process and data processing.

The thermometer resistance and the calorimeter power heating are measured by a potentiometer method with cyclic inversion of the direction of thermometer current for excluding the thermal electromotive forces. All the procedures that control the measurement process are carried out by the PC, which has a simple and user-friendly interface. The results of the measurements are printed and displayed on the screen for visual monitoring. An adiabatic condition in calorimeter is maintained by the AK-9.02 system, which allows keeping the temperature drop between the container and shield on the average within $\pm(1-3) \cdot 10^{-3} \mathrm{~K}$. Owing to modification of the calorimeter, the drop of temperature was reduced to $\sim 0.5 \mathrm{mK}$ at the expense of using an eleven - junctions thermocouple instead of 
four - junction one and employing an additional heater $(R \sim 133 \Omega)$ mounted in the upper part of the shield, to which electrical wires of the thermometer and the main heater were connected. Additional heater allows making up a lack of the second adiabatic shield that is usually employed in the adiabatic calorimeters, but cannot be place in our miniature device. Due to small size, the cryostat with the calorimeter was placed in the transport Dewar vessels with refrigerants (liquid helium or nitrogen), that allows us to exclude an intermediate Dewar vessel and, thus, to keep the coolants. There is no constant pumping of the cryostat during the operation, since high vacuum inside the cryostat was kept by means of cry-sorption provided with an efficient charcoal getter. The degree of vacuum in cryostat is controlled by the value of the heater current in the adiabatic shield. This value was determined in a process of the calorimeter production using nitrogen and helium baths. The automatic procedure of the heat capacity measurements is performed by AK - 9.02 system running under PC control (Pvese \& Malishev, 1994). The program realizes a method of the discrete input of the energy in two modes: constant increments of temperature, $\Delta T$ (from 1 to 2) $\mathrm{K}$ during measurement of the heat capacity and constant impulses of energy in studying the phase transitions.

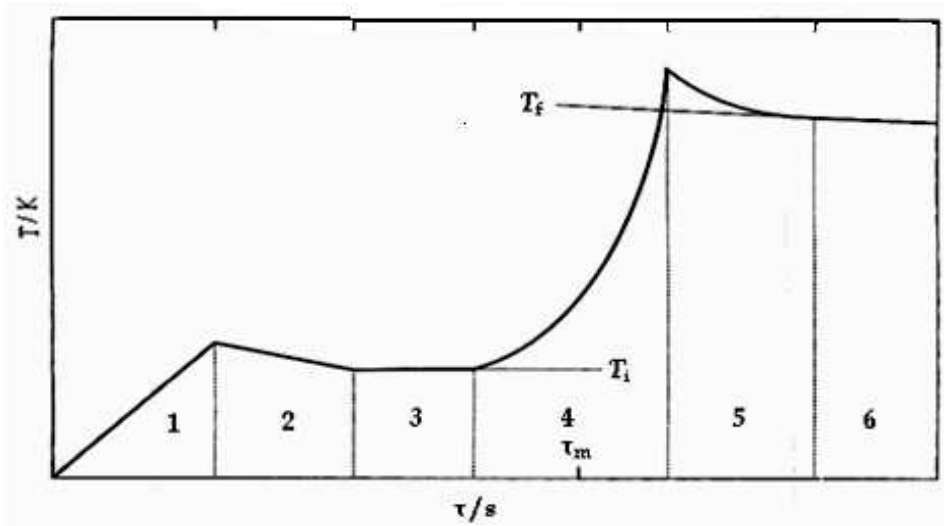

Fig. 6. Temperature, $T$, against time, $\tau$, curve in a heat capacity measurement. 1 to 6 are periods of the calorimetric measurement; $T_{i}$ and $T_{f}$ are an initial and final temperatures of the calorimeter in $4^{\text {th }}$ main (heating) period; $\tau_{\mathrm{m}}$ is the midpoint.

The calorimetric experiment consists of six periods (Fig. 6). In the first period the calorimeter is heated to a desired temperature. A steady temperature equilibration is attained in the second period. In the third period the temperature of the calorimeter is monitored over a chosen time interval to acquire information about the temperature drift rate, $V_{\mathrm{i}}$ and to obtain the linear relation between the values $V_{i}$ and the time by the least-squares method. During the fourth (heating) period the electrical energy is supplied to the calorimeter, and the heating-up time is observed. The fifth period is the same as the second one. In the sixth period the linear relation between the temperature drift rate of the calorimeter $V_{\mathrm{f}}$ and the time is established exactly in a similar manner to that in the third period. The initial and the final temperatures of the calorimeter in the main (heating) period are calculated by 
extrapolating the linear dependencies of the drift rates $V_{\mathrm{i}}$ and $V_{\mathrm{f}}$ on time to the midpoint $\left(\tau_{\mathrm{m}}\right)$ temperature (Fig. 6). This method permits the heat interchange between the calorimeter and surroundings to be taken into account (Varushchenko et al., 1997a). The reliability of this method was proved by a congruence within (0.1 to 0.2$)$ per cent of the heat capacity values of an empty calorimeter, measured in the temperature interval (90 to 110) K using different refrigerants: liquid helium and nitrogen.

The values of the heat capacities, $C_{s, m}$, are fitted with polynomials:

$$
\begin{gathered}
C_{s, m}=\sum_{i} A_{i}\left\{\left(T-A_{k}\right) / B_{k}\right\}^{i} \\
C_{s, m}=\sum_{i} A_{i}^{\prime} \ln \left\{\left(T-A_{k}^{\prime}\right) / B_{k}^{\prime}\right\}^{i}
\end{gathered}
$$

where $\left\{\left(T-A_{k}\right) / B_{k}\right\}$ and $\left\{\left(T-A_{k}^{\prime}\right) / B_{k}^{\prime}\right\}$ are normalizing term, $i$ is degree of polynomials. The coefficients of the polynomials $A_{i}$ and $A_{i}^{\prime}$ were estimated by the LSM.

The metrological characteristics of the calorimeter were tested by measuring the heat capacity of pure copper having a mass fraction of 0.99995 and $n$-heptane in the temperature intervals (from 8 to 372) $\mathrm{K}$ and (from 6 to 354) $\mathrm{K}$, respectively. Obtained $C_{s, m}$ values of copper and $n$-heptane came to an agreement with the precise heat capacities of standard substances within (0.2 to 1.4$) \%$ below the temperature $70 \mathrm{~K}$ and decrease to $(0.01$ and $0.3) \%$ above $T=70 \mathrm{~K}$.

\subsection{Determination of thermodynamic properties of the phase transitions}

The important characteristics of the substances: a triple point temperature, $T_{t p}$, and a mole fraction of impurities, $N_{2}$, were determined by calorimetrical method of the fractional melting study, developed by Mair, Glasgow and Rossini. A linear dependence between the reciprocal fractions of the sample melted, $1 / F_{\mathrm{i}}$, and the equilibrium fusion temperatures, $T_{i}$, makes it possible to calculate both the $T_{t p}$ value and mole fraction of impurities, $N_{2}$ ， by equations:

$$
\begin{gathered}
T_{\mathrm{i}}=d+f \cdot\left(1 / F_{\mathrm{i}}\right), \\
N_{2}=\left(\Delta_{\text {fus }} H_{\mathrm{m}} \cdot f\right) /\left(R \cdot T_{\mathrm{tp}}^{2}\right)
\end{gathered}
$$

Here $d=T_{0}$ is the triple point temperature, $T_{t p}$, of the pure compound, $f=\left(T_{1}-T_{0}\right)$, denotes a depression of the $T_{t p}$ value caused by impurities, $T_{1}$ is the triple point temperature of the completely melted substance (for $F_{\mathrm{i}}=1$ ), $R=8.314472 \mathrm{~J} \mathrm{~K}^{-1} \mathrm{~mol}^{-1}$, and $\Delta_{f u s} H_{m}$ is the enthalpy of fusion, determined by independent method. 
Concave curve of the dependence $T_{\mathrm{i}}=f\left(1 / F_{\mathrm{i}}\right)$ can be explained by formation of solid solution of impurities with main substance. The efficient coefficient of impurities distribution between the crystal and liquid phases $k$ is calculated by Mastrangello's and Dornte's method. The $k$ value close to zero, proves an absence of the solid solution. According to (Alexandrov, 1975), melting curves can be concave not only in the case of solid solutions, but also in the absence of equilibrium in the calorimeter at the onset of fusion, when the amount of the liquid phase is small and impurities can therefore be distributed no uniformly, and at the final stage of melting, when sedimentation of crystals to the bottom of container interferes with slow attainment of temperature equilibrium. In conformity with Alexandrov recommendation (Alexandrov, 1975), the $T_{\text {tp }}$ and $N_{2}$ values ought to be estimated on the basis of the linear dependence for the part of melting curve in the range of $1 / F_{\mathrm{i}}$ values from 1.2 to about 8-10.

In the case of solid solution formation, the mole fraction of impurity, $N_{2}$, can be determined by the Smit and Alexsandrov method using $T_{\mathrm{i}}=f\left(1 / F_{\mathrm{i}}\right)$ experimental data and the equation for $\mathrm{N}_{2}$ in a binary system with solid solution formation (Van Wijk \& Smit, 1960) and (Alexandrov et al., 1983):

$$
T_{i}=T_{0}-\left(N_{2} / A_{k}\right) \cdot\left[(1-k) / F_{i}^{1-k}\right]
$$

Here, $A_{k}$ is cryoscopic constant for the major substance; and $k$ is a distribution coefficient of impurities between the solid and liquid phases. An insufficiency of this equation for calculating $\mathrm{N}_{2}$ consists in the need to determine $k$ coefficient by an independent method. By differentiating and finding the logarithm, equation (15) was transformed by (Alexandrov et al., 1983) to the form:

$$
\ln \left\{-d T_{i} / d\left(1 / F_{i}\right)\right\}=\ln \left\{N_{2} \cdot(1-k)^{2} / A_{k}\right\}-k \cdot \ln \left(1 / F_{i}\right)
$$

Equation (16) is used in this work for determination of the $k$ coefficient directly from experimental dependence of $T_{\mathrm{i}}=f\left(1 / F_{\mathrm{i}}\right)$ and for calculation of the mole fraction, $N_{2}$, of impurity. The $k$ and $N_{2}$ values were calculated by least-squares fits of the $T_{i}$ and $F_{i}$ experimental data using the linear equation (16). Mole fraction of impurity, ${ }^{i} N_{2}$, was computed from the $\ln \left\{N_{2} \cdot(1-k)^{2} / A_{k}\right\}$ term using the $k$ value and the cryoscopic constant $A_{k}=\Delta_{f u s} H_{m}^{0} / R T_{0}^{2}$ determined by above mentioned Rossini method. The enthalpy of fusion $\Delta_{f u s} H_{m}$ is determined by calorimetric method using the total enthalpy absorbed during the fusion with following subtraction of the normal heat capacities of the crystal and liquid which the substance has in the fusion region. The $\Delta_{f u s} H_{m}$ value is obtained from the equation:

$$
\Delta_{\text {fus }} H_{m}=\Delta_{\text {tot }} H-\Delta H_{1}-\Delta H_{2}-\Delta H_{3}
$$

where $\Delta_{t o t} H$ is the total enthalpy absorbed in heating the calorimeter from initial temperature $T_{1}<T_{t p}$ to final one $T_{2}>T_{t p} ; \Delta H_{1}$ and $\Delta H_{2}$ are the changes of enthalpy 
calculated from the normal heat capacities of the crystal and liquid in the temperature intervals from $T_{1}$ to $T_{t p}$ and from $T_{t p}$ to $T_{2}$, respectively; $\Delta H_{e m p}$ is the enthalpy increment need for heating the empty calorimeter from $T_{1}$ to $T_{2}$.

\subsection{Crystal phase transitions and molecular dynamics}

The solid state transitions revealed in the molecular crystals can be explained by different polymorphous transformations, caused by changing the crystal structure, different location of the molecules and their orientational and conformational disorder in the crystal lattice. In this chapter, some thermodynamic properties of solid state transitions and fusion are reviewed for some compounds, which were studied in the Luginin's Thermochemistry Laboratory of the Moscow State University and in some other thermodynamic Laboratories. An interpretation of the solid-state transitions in organic crystals was successfully fulfilled in a set of outstanding researcher's works (Westrum \& McCullough, 1965; Kolesov, 1995; Adachi, et al., 1968) and the others. An interpretation of calorimetric measurements was carried out very often on the basis of the order - disorder concept. Understanding these processes requires sometimes exploring the molecular crystals by X-ray crystallography and IR and Raman spectroscopy. In this work, the solid state transitions will be discussed including some additional physico-chemical properties of the compounds.

The values of thermodynamic properties of the phase transitions are given in Table 5.

It was found by exploring IR-spectra of $\mathrm{CF}_{2} \mathrm{ClCFCl}_{2}$, that this substance comprises a mixture of trans- and gauche- conformers in solid (crystal I) and liquid states (Fig. 7). The sum $\Delta_{t r s} S_{m}^{0}+\Delta_{f u s} S_{m}^{0}=20.52 \mathrm{~J} \cdot \mathrm{K}^{-1} \cdot \mathrm{mol}^{-1}$ for $\mathrm{CF}_{2} \mathrm{ClCFCl}_{2}$ is small, while such sum used to be from (42 to 50 ) $\mathrm{J} \cdot \mathrm{K}^{-1} \cdot \mathrm{mol}^{-1}$ for organic crystals. By comparesing the calorimetric and spectroscopic $S_{m}^{0}$ values, it was found that $\mathrm{CF}_{2} \mathrm{ClCFCl}_{2}$ has residual entropy, $S(0)=10.1 \mathrm{~J} \cdot \mathrm{K}^{-1} \cdot \mathrm{mol}^{-1}$ at $\mathrm{T}=$ 198.15 K (Higgins \& Lielmers, 1965; Kolesov, 1995).
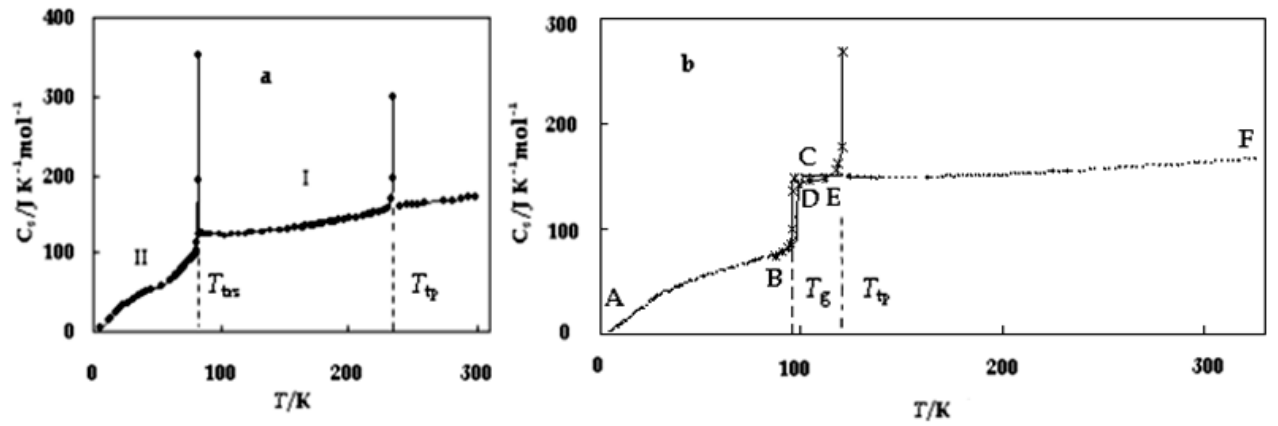

Fig. 7. Heat capacities and phase transitions of $\mathrm{CF}_{2} \mathrm{ClCFCl}_{2}$ (a) and $\mathrm{CF}_{2} \mathrm{ClCHCl}_{2}$ (b). Freon $\mathrm{CF}_{2} \mathrm{ClCHCl}_{2}$ was in following states: glass (AB) and supercooled liquid (CE) $(\alpha=0)$; partially crystalline state (BD) and supercooled liquid (DE) $(\alpha=0.076)$; and liquid (EF) 


\begin{tabular}{|c|c|c|c|c|c|c|}
\hline \multirow{2}{*}{ Compounds } & $T_{\text {trs }}$ & $T_{t p}$ & $\Delta_{t r s} H_{m}^{0}$ & $\Delta_{f u s} H_{m}^{0}$ & $\Delta_{t r s} S_{m}^{0}$ & $\Delta_{f u s} S_{m}^{0}$ \\
\hline & \multicolumn{2}{|c|}{ K } & \multicolumn{2}{|c|}{$\mathrm{kJ} \cdot \mathrm{mol}^{-1}$} & \multicolumn{2}{|c|}{$\mathrm{J} \cdot \mathrm{K}^{-1} \cdot \mathrm{mol}^{-1}$} \\
\hline \multicolumn{7}{|c|}{ Freons } \\
\hline $\mathrm{CF}_{2} \mathrm{ClCHFCl}$ & $86.5 \pm 0.1^{a}$ & $135.74 \pm 0.19$ & - & $7.05 \pm 0.13$ & - & $51.94 \pm 0.71$ \\
\hline $\mathrm{CF}_{2} \mathrm{ClCF}_{2} \mathrm{Cl}^{b}$ & $\begin{array}{l}109.3 \pm 0.2 \\
134.6 \pm 0.1\end{array}$ & $180.62 \pm 0.02$ & $\begin{array}{l}1.21 \pm 0.01 \\
2.63 \pm 0.01\end{array}$ & $1.51 \pm 0.01$ & $\begin{array}{c}11.1 \pm 0.1 \\
19.54 \pm 0.04\end{array}$ & $8.36 \pm 0.04$ \\
\hline $\mathrm{CF}_{2} \mathrm{ClCFCl}_{2} b$ & $82.5 \pm 0.5$ & $236.92 \pm 0.02$ & $0.83 \pm 0.01$ & $2.47 \pm 0.01$ & $10.1 \pm 0.2$ & $10.42 \pm 0.04$ \\
\hline \multicolumn{7}{|c|}{ Derivatives of ferrocene ${ }^{c}$} \\
\hline$n$-PF & $186.7 \pm 0.3$ & $276.83 \pm 0.50$ & $1.23 \pm 0.01$ & $12.74 \pm 0.17$ & $6.59 \pm 0.06$ & $46.02 \pm 0.61$ \\
\hline$i$-BTF & $207.4 \pm 0.3^{a}$ & $294.68 \pm 0.40$ & - & $15.05 \pm 0.08$ & - & $51.07 \pm 0.27$ \\
\hline $\mathrm{BeF}$ & $\begin{array}{l}44.3 \pm 0.2 \\
51.1 \pm 0.3\end{array}$ & $349.57 \pm 0.10$ & $\begin{array}{l}23.8 \pm 0.2 \\
46.1 \pm 0.4\end{array}$ & $25.54 \pm 0.94$ & $\begin{array}{l}0.54 \pm 0.01 \\
0.83 \pm 0.01\end{array}$ & $73.06 \pm 2.70$ \\
\hline POF & - & $311.62 \pm 0.51$ & - & $19.16 \pm 0.12$ & - & $61.48 \pm 0.39$ \\
\hline$i$-BF & - & $279.96 \pm 0.10$ & - & $15.33 \pm 0.04$ & - & $54.76 \pm 0.14$ \\
\hline $\mathrm{BeOF}^{d}$ & - & 380.7 & - & 29.9 & - & 78.5 \\
\hline FM & - & $347.80 \pm 1.0$ & - & $22.91 \pm 0.53$ & - & $65.87 \pm 1.52$ \\
\hline \multicolumn{7}{|c|}{ Alkylderivatives of adamantane } \\
\hline 1,3,5-TMA & $234.4 \pm 0.1$ & $255.61 \pm 0.05$ & $8.19 \pm 0.03$ & $2.06 \pm 0.03$ & $34.94 \pm 0.13$ & $8.06 \pm 0.11$ \\
\hline 1,3-DMA & $223.38 \pm 0.01$ & $247.79 \pm 0.01$ & $9.31 \pm 0.01$ & $1.54 \pm 0.01$ & $41.68 \pm 0.06$ & $6.21 \pm 0.02$ \\
\hline 1-EA & - & $225.56 \pm 0.02$ & - & $11.22 \pm 0.03$ & - & $49.74 \pm 0.08$ \\
\hline \multicolumn{7}{|c|}{ Bicyclic hydrocarbons } \\
\hline cis- $\mathrm{C}_{9} \mathrm{H}_{16}$ & $\begin{array}{c}182.28 \\
184.9\end{array}$ & 236.48 & $\begin{array}{l}8.26 \pm 0.004 \\
0.40 \pm 0.002\end{array}$ & $1.40 \pm 0.004$ & $\begin{array}{l}45.3 \\
2.2\end{array}$ & 5.92 \\
\hline trans $-\mathrm{C}_{9} \mathrm{H}_{16}$ & - & 213.86 & - & $10.90 \pm 0.02$ & - & 50.97 \\
\hline cis- $\mathrm{C}_{10} \mathrm{H}_{18}$ & 216.1 & $230.18 \pm 0.05$ & 2.136 & $9.489 \pm 0.006$ & 9.88 & 41.22 \\
\hline trans $-\mathrm{C}_{10} \mathrm{H}_{18}$ & & $242.78 \pm 0.05$ & & $14.414 \pm 0.001$ & & 59.37 \\
\hline \multicolumn{7}{|c|}{ Bicyclic perfluorocarbons } \\
\hline cis- $\mathrm{C}_{9} \mathrm{~F}_{16}$ & \begin{tabular}{|l|}
$200.64 \pm 0.03$ \\
$245.63 \pm 0.04$
\end{tabular} & $291.27 \pm 0.09$ & \begin{tabular}{|c|}
$8.76 \pm 0.03$ \\
1.27
\end{tabular} & $2.72 \pm 0.03$ & \begin{tabular}{|c|}
$43.66 \pm 0.04$ \\
5.17
\end{tabular} & $9.34 \pm 0.09$ \\
\hline trans- $\mathrm{C}_{9} \mathrm{~F}_{16}$ & $236.63 \pm 0.02$ & $248.05 \pm 0.04$ & 8.90 & $2.63 \pm 0.02$ & 37.6 & $10.60 \pm 0.10$ \\
\hline cis- $\mathrm{C}_{10} \mathrm{~F}_{18}$ & $232.5 \pm 0.2$ & $266.95 \pm 0.02$ & $4.24 \pm 0.01$ & $10.30 \pm 0.01$ & $18.24 \pm 0.06$ & $38.58 \pm 0.15$ \\
\hline trans $-\mathrm{C}_{10} \mathrm{~F}_{18}$ & - & $294.83 \pm 0.02$ & - & $17.96 \pm 0.04$ & - & $60.92 \pm 0.14$ \\
\hline $\mathrm{C}_{5} \mathrm{~F}_{10} \mathrm{~N}-\mathrm{C}_{6} \mathrm{~F}_{10}-\mathrm{CF}_{3}$ & - & $293.26 \pm 0.20$ & - & $8.32 \pm 0.02$ & - & $28.37 \pm 0.06$ \\
\hline
\end{tabular}

${ }^{a}$ Glass like transition, $T_{g} ;{ }^{b}$ Kolesov, 1995) ${ }^{c} n$-propylferrocene [n-PF], iso-butyrylferrocene [i-BTF], benzylferrocene $[\mathrm{BeF}]$, propionylferrocene $[\mathrm{POF}]$, iso-butylferrocene $[i-\mathrm{BF}]$, benzoylferrocene $[\mathrm{BeOF}]$, ferrocenylmethanol $[\mathrm{FM}]{ }^{;}{ }^{d}$ values were measured by DSC.

Table 5. The temperatures, $T_{t r s}, T_{t p}$, enthalpies, $\Delta_{t r s} H_{m}^{0}, \Delta_{f u s} H_{m}^{0}$, and entropies, $\Delta_{t r s} S_{m}^{0}, \Delta_{f u s} S_{m}^{0}$ of the solid-to-solid transitions and fusions, respectively, for some freons, ferrocene derivatives, cyclic hydrocarbons, and perfluorocarbons. 
A characteristic feature of solid state transition of organic crystals is a slow thermal equilibrium between co-existing phases which very often promote to formation of metastable phases existing in a wide temperature range. In Fig. 7(b), the heat capacity $C_{S, m}$ of 1,1-difluoro-1,2,2-trichloroethane, $\mathrm{CF}_{2} \mathrm{ClCHCl}_{2}$, is shown in the temperature interval studied. Similar $C_{S, m}=f(T)$ dependence has been obtained for isomer of 1,1-difluoro-1,2,2trichloroethane: $\mathrm{CFCl}_{2} \mathrm{CHFCl}$. Both isomers were in the forms of glasses, supercooled liquids, and partially crystalline states. The latter was attained after annealing the specimen at temperatures from (110 to 114) $\mathrm{K}$ during 3 days, followed by quenching it at $T \approx 78 \mathrm{~K}$ over a period of $12 \mathrm{~h}$. The heat capacity jumps, accompanying G-transitions, are observed on the $C_{S}-T$ curves of both freons. Taking into account this typical transitions for the glasses, the authors of reference (Adachi, et al., 1968) proposed a term "glassy crystal" for the frozen - in disordered states (AB) (Fig.7, (b)). The temperatures of the glass transition $T_{g}=95.7 \mathrm{~K}$ and fusion, $T_{f u s}=123.1 \pm 0.4 \mathrm{~K}$ have been obtained. The degrees of crystallinity, $\alpha$, appropriated to the mole fraction of crystalline samples, equal to 0.076 and 0.116 for $\mathrm{CF}_{2} \mathrm{ClCHCl}_{2}$ and $\mathrm{CFCl}_{2} \mathrm{CHFC}$, respectively, were calculated on the basis of calorimetric data on $\Delta C_{S}$ jumps by studying the G-transitions (Varushchenko et al., 1997b).

Fig. 8 presents the $C_{S}-T$ curve of ferrocenyl- $n$-propane, which exhibits a fusion and a gradual solid-to-solid transition in the temperature range from (156 to 204) K. The temperature of the gradual transition of crystal II to crystal I of ferrocenyl- $n$-propane was ascribed to that of the maximum $C_{S}$-value in the peak of solid state transition. A test of the calorimetric experiment showed that $\lambda$-anomaly was accompanied by decreasing the heat capacity, $\Delta C_{S}=-2.7 \mathrm{~J} \cdot \mathrm{K}^{-1} \cdot \mathrm{mol}^{-1}$ and continuance changing the enthalpy and entropy. Thus, the solid-state anomaly is the phase transition of the second order and can be interpreted as the "order-disorder" transformation. The changes of the enthalpy, $\Delta_{t r s} H_{m}^{0}$, and entropy, ${ }_{t r s} S_{m}^{0}$ of the thermal anomaly were evaluated by summing up these values in each experimental $C_{S}$ point with subtracting changes of appropriate functions for the empty calorimeter and those ones for the hypothetic normal parts of the crystals I and II.

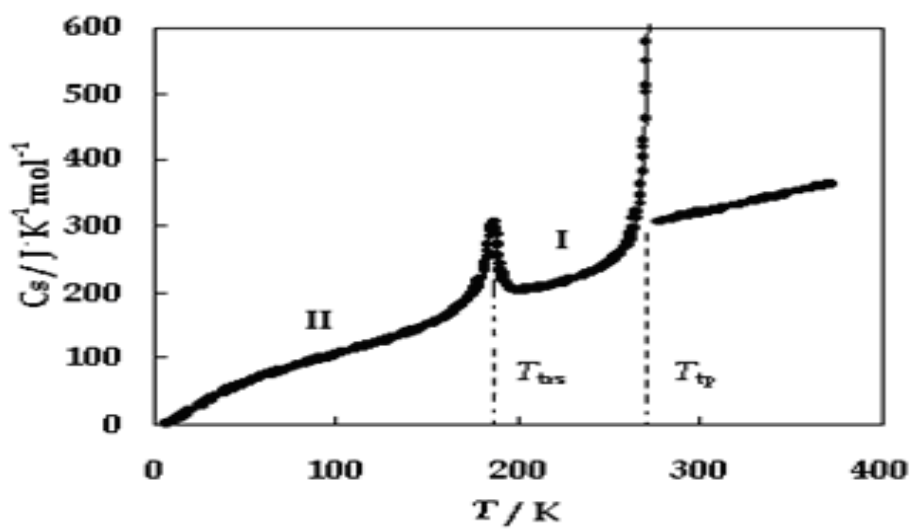

Fig. 8. Molar heat capacity, $C_{S}$, of ferrocenyl- $n$-propane as a function of temperature, $T$, where $T_{t p}$, and $T_{t r s}$ denote the triple point and temperature of $\lambda$-like transition. 
The nature of this transition was studied by the X-ray crystallography. Table 6 lists crystallographic data of $n$-propylferrocene in vicinity of the "order-disorder" transition. The structure of $n$-propylferrocene at $200 \mathrm{~K}$ contains a propyl- group disordered between two positions (in ca. 2:1 ratio) obviously due to thermal motion (Fig. 9). The transition of the crystal II $(T=150 \mathrm{~K})$ to the crystal I $(T=200 \mathrm{~K})$ occurred with significant changes of the lattice parameters: basis vectors, $a, b, c$, angle, $\beta$, the volume, $V$, the number of molecule in the unit cell, $Z$, and the factors of the crystal structure quality, $R_{1}$ and $w R_{2}$.
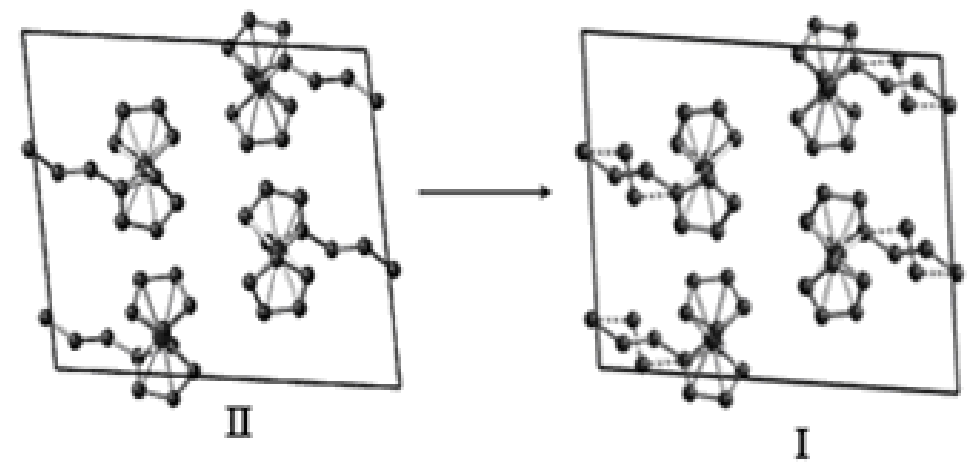

Fig. 9. The structure of the ferrocenyl-n-propane crystal at the temperatures $150 \mathrm{~K}$ (II) and $200 \mathrm{~K}(\mathrm{I})$

\begin{tabular}{|c|c|c|c|c|c|c|c|c|c|}
\hline $\mathrm{T} / \mathrm{K}$ & $\begin{array}{c}\text { symmetry, } \\
\text { space } \\
\text { group }\end{array}$ & $10^{-10} a / \mathrm{m}$ & $10^{-10} b / \mathrm{m}$ & $10^{-10} c / m$ & $\beta / 0$ & $10^{-30} \mathrm{~V} / \mathrm{m}^{3}$ & Z & $R_{1}$ & $w R_{2}$ \\
\hline 150 & \multirow{2}{*}{$\begin{array}{c}\text { monoclinic } \\
P 2_{1} / c\end{array}$} & $13.738(4)$ & $7.541(1)$ & $10.660(2)$ & $99.76(3)$ & 1088.4(4) & \multirow[b]{2}{*}{4} & 0.061 & 0.112 \\
\hline 200 & & $13.977(4)$ & $7.621(1)$ & $10.521(2)$ & $96.87(3)$ & $1112.6(4)$ & & 0.064 & 0.136 \\
\hline
\end{tabular}

Table 6. Crystallographic data of $n$-propylferrocene crystal at the temperatures $150 \mathrm{~K}$ and $200 \mathrm{~K}$

While both $a$ and $b$ parameters became larger during phase transition II to I, the $c$ parameter and $\beta$ angle slightly decreased (Table 6). Structure solution of the crystals I and II revealed that lower temperature modification of the n-propylferrocene molecule has only one orientation of the propyl group. Apparently, the transitions in reverse order occurred when cooling the crystals from (150 to 200$) \mathrm{K}$. Thus, the solid-state anomaly of $n$-propylferrocene is caused by the onset of the internal rotation of propyl groups in the molecules and also by a small shift of the pentadienyl cycles around the axis passed through their centers. These variations led to some orientation disorder of the crystal phase II (Fig. 9).

The order-disorder conception is successfully used in exploring the plastic crystals. Adamantane and some of its derivatives form disordered plastic crystalline phases. The fusion of such substances occurs some times in two stages. First, an orientational disorder proceeds in the crystalline lattice because of high mobility of the molecules, and then the plastic crystals fuse owing to a translational molecular motion at higher temperature. In this 
case, the magnitudes of enthalpy and entropy of the solid-to-solid transition are several times larger than those of the fusion. In accordance with the empirical Timmerman's criterion, the $\Delta_{f u s} S_{m}^{0}$ values for the plastic crystals are usually less than $20 \mathrm{~J} \cdot \mathrm{mol}^{-1} \cdot \mathrm{K}^{-1}$. There are two modifications of the plastic crystals with different molecular reorientations, isotropic and anisotropic. Some substances are known to form both of reorientations.

Fig. 10 presents $C_{S}-T$ curve of 1,3,5-trimethyladamantane (1,3,5-TMA) explored in this work (a) and the Raman spectrum of the substance (b). A spectroscopic investigation of 1,3,5-TMA was carried out together with that of 1,3-dimethyladamantane (1,3-DMA), which also formed plastic crystals. Both compounds have low values of the entropy of fusion, $\Delta_{\text {fus }} S_{m}^{0}=8.1$ (6.2) $\mathrm{J} \cdot \mathrm{K}^{-1} \cdot \mathrm{mol}^{-1}$ and narrow temperature intervals of existence of the high temperature crystal I, $\Delta T=21(24.2) \mathrm{K}$, respectively. According to adiabatic calorimetry, these properties are typical for the plastic crystals. Distinct bands were observed in their spectra at the low temperatures. As it follows from joint spectra discussion, the number of bands for 1,3,5-TMA is about haft of that for the 1,3-DMA crystal. This suggests that more symmetric molecules of the former compound compose the lattice of higher symmetry and/or with the less quantity of units in a primitive cell. In the vicinity of the phase transitions, the changes in spectra become more pronounced. At the transition points, all the bands disappear and transform into the wing of a broad Rayleigh scattering. This implies that all of them were caused by the librational modes in the low-temperature crystals of 1,3- DMA and 1,3,5- TMA and that the character of molecular motion is different in high-temperature solid phases. The absence of the preferential axes of molecular reorientation in the latter implies that there are isotropic plastic crystals. Analysis of Raman

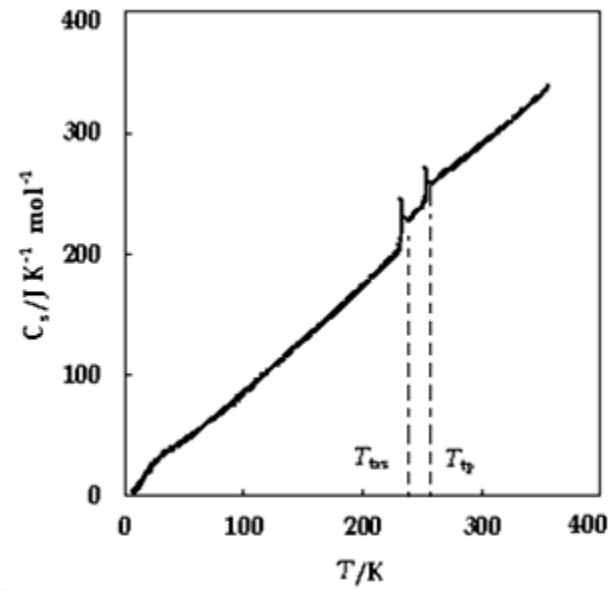

a

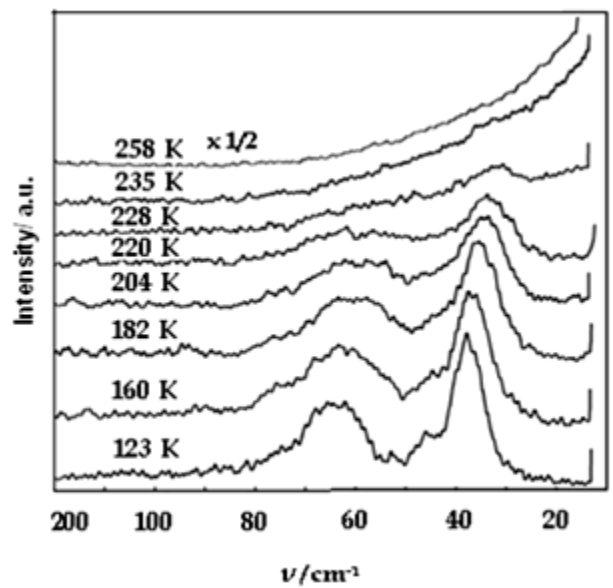

b

Fig. 10. (a) The heat capacity of 1,3,5-trimethyladamantane in dependence on temperature, $T$, where $T_{t p}$, and $T_{t r s}$ denote temperatures of the triple point and the solid-to-solid transition. (b) The temperature dependence of low-frequency Raman spectrum of 1,3,5trimethyladamantane. 
spectra is in agreement with the low values of transition entropies measured by adiabatic calorimetry for alkylderivatives of adamantane. The cis- and trans- isomers of perfluorobicyclo $(4,3,0)$ nonane are almost spherical "globular" molecules, which are able to form the plastic crystals due to unusually high molecular mobility. The thermodynamic properties of compounds are known to change when going from fluoroorganic compounds to their hydrogen containing analogous. With this in mind, the thermodynamic properties of the solid state transitions of perfluorocarbons are compared with those of appropriate hydrocarbons.

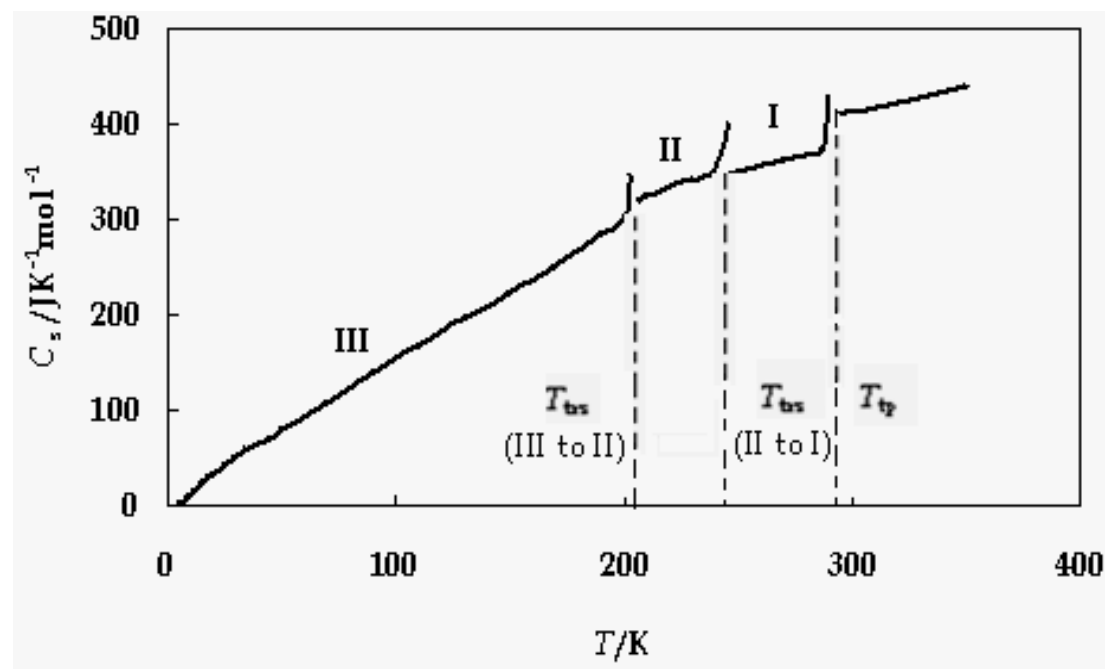

Fig. 11. Molar heat capacity, $C_{S}$, of cis-perfluorobicyclo(4,3,0)nonane as function of temperature, $T$, where $T_{\text {trs }}$ (III to II) and $T_{\text {trs }}$ (II to I) denote the temperatures of the solid-tosolid transitions and $T_{t p}$ is the temperature of triple point

Fig. 11 presents a temperature dependence of the heat capacity of cisperfluorobicyclo $(4,3,0)$ nonane. Two solid states transitions can be interpreted as the orderdisorder transformation caused by orientation disorder. Taking into account the high entropy values of $\Delta_{t r s} S_{m}^{0}=43.66 \pm 0.04 \mathrm{~J} \cdot \mathrm{mol}^{-1} \cdot \mathrm{K}^{-1}$ (Table 5) for transition of the crystal III-to- crystal II, this solid phase conversion can be attributed to an anisotropic molecular reorientation about preferential common C-C axis. According to the empirical Timmerman's criterion, small values of the fusion entropy, ${ }{ }_{f u s} s_{m}^{0}=9.34 \pm 0.09 \mathrm{~J} \cdot \mathrm{mol}^{-1} \cdot \mathrm{K}^{-1}$ of $c i s-\mathrm{C}_{9} \mathrm{~F}_{16}$ (Table 5) indicates the onset of the isotropic molecular reorientation and, thus the formation of the plastic crystals.

As is seen from Table 5, the cis-bicyclo $(4,3,0)$ nonane has thermodynamic properties of the solid-state transition, analogous to its perfluorated counterpart, and forms plastic crystals. The trans-perfluorobicyclo(4,3,0)nonane exhibits one solid-state transition and also forms the plastic crystals, but its hydrogen analogous, trans-bicyclo $(4,3,0)$ nonane do not form the plastic crystals. These properties of the trans-isomers can be explained by their less spherical shapes as compared with the cis-isomers. 


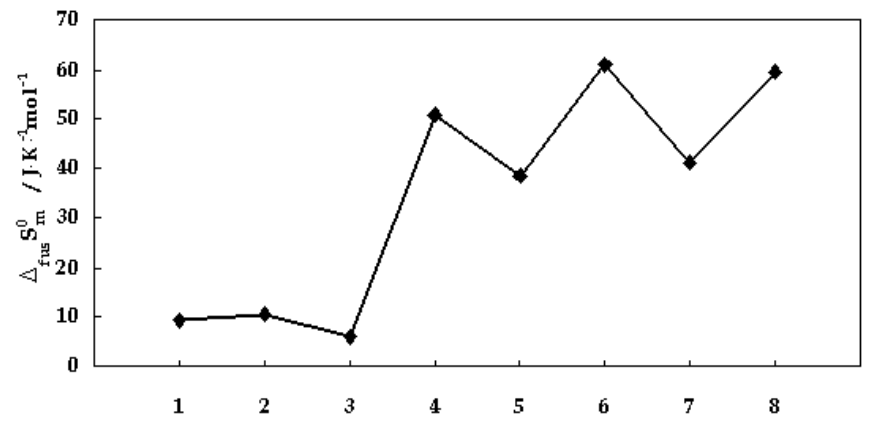

Fig. 12. Molar entropies of fusion $\Delta_{f u s} S_{m}^{0}$ for some bicyclic perfluorocarbons and appropriate hydrocarbons: cis- [1] and trans- [2] perfluorobicyclo $(4,3,0)$ nonanes; cis- [3] and trans- [4] bicyclo $(4,3,0)$ nonanes; cis- [5] and trans- [6] perfluorobicyclo $(4,4,0)$ decanes; cis- $[7]$ and trans- [8] bicyclo $(4,4,0)$ decanes

Analysis of the entropies of fusion for cis- and trans-isomers of perfluorobicyclo $(4,3,0)$ nonanes and $-(4,4,0)$ decanes and appropriate values of hydrogen - containing analogous (Table 5, Fig. 12) permits to interpret the influence of the structure and chemical nature on molecular mobility and thermodynamic properties of the solids. Comparesing the entropies of fusion shows that the mobility of the molecules increases in going from hydrocarbons to appropriate perfluorocarbons and from the cis-isomers to the trans-ones. The larger molecular mobility of the perfluorocarbons can be explained by more weak intermolecular interactions for these compounds compared with the hydrocarbons. The greater ability of the molecules of cis-isomer to reorient in the solid state seems to be due to the steric factors. The nature of the solid-to-solid transitions in cis- [7] and trans- [8] bicyclodecanes and cis- [5] and trans- [6] perfluorobicyclodecanes (Fig. 12) were discussed in the order-disorder concept in reference (Kolesov, 1995).

\section{Thermodynamic functions in the ideal gas states}

The absolute entropy, changes of the enthalpy and Gibbs energy in three aggregate states are calculated on the basis of smoothed heat capacity values. The experimental data on the heat capacities of the substances under study in the temperature intervals from (6/8 to 372) $\mathrm{K}$ were fitted by polynomial (11). Extending the heat capacities to $T \rightarrow 0$ were performed by Debye equation:

$$
C_{\mathrm{S}, \mathrm{m}}=n D(\Theta / T)
$$

where $D$ is the Debye function, $n=3$, and $\Theta$ denote Debye characteristic temperature.

Testing the $C_{\mathrm{s}, \mathrm{m}}$ values at $T \rightarrow 0$ was performed by fitting the heat capacities in small temperature interval below (10 to 12 ) K by equation:

$$
C_{s, m} / T=\alpha T^{2}+\gamma,
$$

where $\alpha$ and $\gamma$ are coefficients. If $\gamma=0$, it can be accepted that $C_{\mathrm{s}, \mathrm{m}}=0$ and extrapolation of the heat capacity to $T \rightarrow 0$ can be carried out by equation (18) or by Debye cube's law, 
$C_{S, m}=A T^{3}$. But, in the case $\gamma \neq 0$, appropriate extending of the heat capacity could be conducted by (18), provided the parameters $n$ and $\Theta$ are adjusted thus to allow one to obtain zero $C_{S, m}$ values at $T=0 \mathrm{~K}$. The $C_{p, m}-C_{S, m}=T(\partial V / \partial T)_{p}(\partial p / \partial T)_{s}$ difference evaluated at $T=298.15 \mathrm{~K}$ was smaller than uncertainties of $C_{S, m}$ for the substances under study and was not taken into account. The smoothed values of $C_{p, m}$ and thermodynamic functions $S_{m}^{O}(T),\left\{H_{m}^{0}(T)-H_{m}^{0}(0)\right\}$, and $\left\{G_{m}^{O}(T)-H_{m}^{0}(0)\right\}$ for the condensed states were calculated by numerical integrating the $C_{p, m}=f(T)$ functions obtained by equations (18) and (11) and adding the enthalpies and entropies of the solid-to-solid transition and fusion. The errors of thermodynamic functions were estimated by the law of random errors accumulation using the uncertainties of the heat capacity measurements. The ideal gas absolute entropy, $S_{m}^{O}(T)$, the changes of the enthalpy and the free Gibbs energy at $298.15 \mathrm{~K}$ were calculated using the appropriate functions in the liquid state, enthalpies and entropy of vaporization and the entropy of the ideal gas compression, $\Delta S=R \cdot \ln \{p(T) /(101.325) \mathrm{kPa}\}$ calculated from the vapor pressure data.

\subsection{Theoretical calculations of the thermodynamic functions}

The ideal gas absolute entropy and heat capacity were calculated by statistical thermodynamics, additive principle (Poling et al., 2001; Domalski et al., 1993; Sabbe et al., 2008), and empirical difference method of group equations (Cohen \& Benson, 1993).

The statistical thermodynamic method was used with quantum mechanical [QM] calculation on the basis of the density functional theory [DFT]. The QM calculation was performed on the level B3LYP/6-31G(d,p) using the Gaussian 98 and 03 software packages (Frisch et al., 2003). As a result, the following constants can be calculated: the moments of inertia of the entire molecule, the moments of inertia for internal rotors, the normal vibrational frequencies, and the barrier to internal rotation. The potential functions of internal rotation were determined by scanning the torsion angles from (0 to 360 $)^{\circ}$ at $10^{\circ}$ increments and allowing all other structural parameters to be optimized at the same level with the subsequent frequency calculation. The calculated potential energies were fitted to the cosine-based Fourier series:

$$
V(\varphi)=V_{0}+0.5 \sum_{n} V_{n}(1-\cos n \varphi),
$$

where $V(\varphi)$ denotes potential energy function, $\varphi$ is torsional angle.

The ideal gas entropies and heat capacities in dependence on the temperature were calculated by standard statistical thermodynamics formulae using the rigid-rotor harmonic oscillator [RRHO] approximation. To account for the internal rotation processes, the torsional frequencies were omitted in the calculation of thermodynamic function. A contribution of the internal rotation for each rotor was calculated by direct summation over the energy levels obtained by diagonalization of the one-dimensional Hamiltonian matrix associated with potential function from equation (20). The RRHO approximation, is known, results in overestimated entropy values for flexible molecules due to coupling the internal rotations. One-dimensional hindered rotor correction has been applied by (Vansteenkiste et al., 2003; Van Speybroeck et al., 2000) assuming decoupled internal rotations.

The method of group equation is suitable for calculation of some additive properties of a compound, namely $S_{m}^{\circ}(T), C_{p, m}^{\circ}(T), \Delta_{f} H_{m}^{o}$ values on the basis of reliable appropriate 
properties of the structurally similar compounds. For example, determination of $S_{m}^{\circ}(T)$ value of ferrocenylmethanol using absolute entropies of the components of hypothetical reaction:

$$
\mathrm{Fe}\left(\mathrm{C}_{5} \mathrm{H}_{5}\right)\left(\mathrm{C}_{5} \mathrm{H}_{4}-\mathrm{CO}-\mathrm{H}\right)=\left[\mathrm{Fe}\left(\mathrm{C}_{5} \mathrm{H}_{5}\right)_{2}\right]+\left[\mathrm{C}_{6} \mathrm{H}_{5}-\mathrm{CO}-\mathrm{H}\right]-\left[\mathrm{C}_{6} \mathrm{H}_{6}\right]
$$

The calculation of the absolute entropy by additive methods requires taking into account corrections for the symmetry and the optical isomerism of the molecule. Otherwise, the principle of group additivity can be broken if these parameters alter when changing the structure of the molecules in the series of compounds. Therefore, an additive calculation of the absolute entropy, $S_{\mathrm{m}}^{0}$, is conducted by using so-called intrinsic entropy, $S_{\mathrm{m}}^{0}$,int , which allows to exclude an influence of the rotary components, depending on the symmetry and optical activity of the molecule:

$$
S_{\mathrm{m}, \text { int }}^{0}=S_{\mathrm{m}}^{0}+R \cdot \ln \left(\sigma_{\mathrm{tot}} / n\right)
$$

where $R \cdot \ln \left(\sigma_{\text {tot }} / n\right)$ is the correction for the symmetry and the chirality of the molecule, $\sigma_{\text {tot }}$ and $n$ denote a total number of the symmetry and the number of the optical isomers. The total symmetry of the molecule is calculated as $\sigma_{\text {tot }}=\sigma_{\mathrm{ex}} \cdot \sigma_{\mathrm{in}}$, where $\sigma_{\mathrm{ex}}$ is the number of symmetry of the external rotation of the molecule, as a whole, and $\sigma_{\text {in }}$ denotes the number of the internal rotation equal to the product $(P)$ of the order of independent axes in the rotating group (l) raised to the power of the number of these axes $(\mathrm{k}), \sigma_{\text {in }}=P \cdot \mathrm{l}^{\mathrm{k}}$. Thus, an additive calculation of the absolute entropy by group equation method requires of a missing intrinsic entropy $S_{\mathrm{m} \text {,int }}^{0}$ determination and following recalculation it to the $S_{\mathrm{m}}^{0}$ value by formula (20).

Comparing between the values of absolute entropy determined on the basis of calorimetric measurements, $S_{m}^{O}(g)($ expt $)$, group equation method, and statistical thermodynamic calculations, $S_{m}^{O}($ stat $)$, are applied for prediction of the missing data, verification of their reliability, and mutual congruence in the series of the same type compounds, or homologous. Below, a critical analysis of the ideal gas entropies is performed for some series of functional organic compounds.

Freons and cloroalkanes. Table 7 lists the ideal gas absolute entropies determined on the basis of experimental data and those ones obtained by theoretical calculation for some freons [R] and cloroethanes [ClE]. The $S_{m}^{o}(g)($ expt $)$ and $S_{m}^{o}($ stat $)$ values for halogenethanes and freons, including $S_{m}^{O}(g)$ values with marks $(a$ and $b)$, agree within errors limits: from (0.1 to 1.3$) \%$. These compounds have rather compact and symmetric molecules and exist as an equilibrium mixture of trans- and gauche- conformers in three aggregate states and as single more stable conformers in the low-temperature crystal phase. There are disagreements between $S_{m}^{O}(g)($ expt $)$ and $S_{m}^{O}(s t a t)$ values in a group of three freons $\mathrm{CF}_{2} \mathrm{ClCFCl}_{2}$ [R-113], $\mathrm{CFCl}_{2} \mathrm{CHFCl}$ [R-122a], and $\mathrm{CF}_{2} \mathrm{ClCHCl}_{2}$ [R-122]. A characteristic feature of these compounds is availability of residual entropies caused by orientational or conformational disorders. The mixtures of the trans- and two gauche-conformers of these freons were probably frozen at low helium temperatures. The entropy change caused by disorder of this type can be evaluated by the formula $\Delta S=R \ln \left(N_{2} / N_{1}\right)$, where $N_{2}$ and 
$N$ are the number of states of disordered and ordered phases. Thus, the residual entropy of $\mathrm{CFCl}_{2} \mathrm{CHFCl}$ and $\mathrm{CF}_{2} \mathrm{ClCHCl}_{2}$ is $S_{m}^{o}(0)=R \cdot \ln (3)=9.1 \mathrm{~J}^{-1} \cdot \mathrm{mol}^{-1}$. The value $S_{m}^{o}(0)=10.1$ $\mathrm{J} \cdot \mathrm{K}^{-1} \mathrm{~mol}^{-1}$ of $\mathrm{CF}_{2} \mathrm{ClCFCl}_{2}$, obtained in (Higgins \& Lielmers, 1965), is in good agreement with the $R \cdot \ln (3)$ value. After taking into account residual entropies of these freons, the calorimetric and theoretical values of absolute entropies agree within errors limits from $(0.1$ to 1.3$) \%$. At the same time, testing the low-temperature $C_{p, m}$ values of $\mathrm{CFCl}_{2} \mathrm{CHFCl}$ and $\mathrm{CF}_{2} \mathrm{ClCHCl}_{2}$ by equation (19) showed an absence of the residual entropies for these freons. Their heat capacities in the temperature interval from (5 to 8) K obeyed Debye cub's law with experimental error of $C_{p, m}$ value, $2 \%$. A disagreement of two methods evaluation of the residual entropy can not be explained on the basis of available physico-chemical data of these freons.

\begin{tabular}{|c|c|c|c|c|c|c|c|c|c|c|c|c|}
\hline $\begin{array}{l}\text { Comp } \\
\text { ounds }\end{array}$ & R-112 & R-113 & R-114 & R-122 & R-122a & R-123 & $\begin{array}{c}\text { R- } \\
123 a\end{array}$ & R-243 & $\begin{array}{c}\text { R- } \\
253 f a\end{array}$ & $\begin{array}{c}1,1- \\
\text { DClE }\end{array}$ & $\begin{array}{c}1,2- \\
\text { DClE }\end{array}$ & $\begin{array}{c}1,1,1- \\
\text { TCIE }\end{array}$ \\
\hline $\begin{array}{c}S_{m}^{o}(g) \\
\left(\begin{array}{l}\text { expt }), \\
\mathrm{J}^{-1} \mathrm{~mol}^{-1}\end{array}\right.\end{array}$ & 382.3 & $\begin{array}{c}378.9 \\
382.0^{a}\end{array}$ & 363.8 & $\begin{array}{c}363.1 \\
372.2^{b}\end{array}$ & $\begin{array}{c}366.2 \\
375.4^{b}\end{array}$ & 352.4 & 365.6 & 394.7 & 365.3 & 304.4 & 307.8 & 321.0 \\
\hline $\begin{array}{c}S_{m}^{o}(\text { stat }) \\
\mathrm{J}^{-1} \mathrm{~mol}^{-1}\end{array}$ & 385.7 & 386.9 & 364.2 & 372.5 & 378.6 & 352.6 & 368.1 & 399.1 & 367.1 & 305.2 & 306.0 & 320.1 \\
\hline
\end{tabular}

$a, b$ Absolute entropies were calculated as $S_{m}^{O}(g)(\operatorname{expt})+S_{m}^{O}(0)$, were $S_{m}^{O}(0) \quad a=10.1 \quad \mathrm{~J}^{-1} \mathrm{~K}^{-1} \mathrm{~mol}^{-1}$ and $S_{m}^{O}(0){ }^{b}=9.1 \mathrm{~J} \cdot \mathrm{K}^{-1} \mathrm{~mol}^{-1}$.

Table 7. The absolute entropies at $T=298.15 \mathrm{~K}$, calculated by statistical thermodynamic, $S_{m}^{o}$ (stat), and determined from the calorimetric data on the heat capacity, entropy of vaporization, and the entropy of the ideal gas compression, $S_{m}^{o}(g)($ expt $)$ for some freons and cloroethanes

\begin{tabular}{|c|c|c|c|c|c|c|c|c|c|c|}
\hline \multirow{2}{*}{ Compounds } & \multicolumn{2}{|c|}{$\mathrm{C}_{9} \mathrm{~F}_{16}$} & \multicolumn{2}{|c|}{$\mathrm{C}_{9} \mathrm{H}_{16}$} & $\mathrm{C}_{10} \mathrm{~F}_{18}$ & \multicolumn{2}{|c|}{$\mathrm{C}_{10} \mathrm{H}_{18}$} & \multirow{2}{*}{$\begin{array}{c}1,3- \\
\text { DMA }\end{array}$} & \multirow{2}{*}{$\begin{array}{l}1,3,5- \\
\text { TMA }\end{array}$} & \multirow{2}{*}{$\begin{array}{l}1- \\
\text { EA }\end{array}$} \\
\hline & cis- & trans- & cis- & trans- & cis- & cis- & trans- & & & \\
\hline $\begin{array}{c}S_{m}^{O}(g)(\text { expt }) \\
\mathrm{J} \cdot \mathrm{K}^{-1} \mathrm{~mol}^{-1}\end{array}$ & $\begin{array}{c}605.3 \\
\pm 3.0 \\
\end{array}$ & $\begin{array}{c}602.8 \\
\pm 3.0 \\
\end{array}$ & 374.20 & 365.39 & $\begin{array}{c}630.7 \\
\pm 0.3 \\
\end{array}$ & 378.86 & 374.43 & $\begin{array}{c}392.9 \\
\pm 1.0 \\
\end{array}$ & $\begin{array}{c}415.2 \\
\pm 1.0 \\
\end{array}$ & $\begin{array}{c}400.9 \\
\pm 1.0 \\
\end{array}$ \\
\hline $\begin{array}{c}S_{m}^{O}(\text { stat }), \\
\mathrm{J} \cdot \mathrm{K}^{-1} \mathrm{~mol}^{-1}\end{array}$ & 610.83 & 606.41 & $377.32^{a}$ & $365.30^{a}$ & 633.33 & 378.81 & 373.81 & 391.4 & 413.4 & 400.0 \\
\hline
\end{tabular}

a Values were taken from (Frenkel et al., 1994).

Table 8 . The absolute entropies at $T=298.15 \mathrm{~K}$, calculated by statistical thermodynamics, $S_{m}^{o}$ (stat), and determined from the calorimetric data on the heat capacity, entropy of vaporization, and the entropy of the ideal gas compression, $S_{m}^{O}(g)($ expt $)$ for cis- and transperfluorobicyclo $(4,3,0)$ nonanes [cis- and trans- $\mathrm{C}_{9} \mathrm{~F}_{16}$ ], cis- and trans- bicyclo $(4,3,0)$ nonane, [cis- and trans- $\mathrm{C}_{9} \mathrm{H}_{16}$ ], cis-perfluorobicyclo $(4,4,0)$ decane, [cis- $\left.\mathrm{C}_{10} \mathrm{~F}_{18}\right]$, cis- and transbicyclo $(4,4,0)$ decanes [cis- and trans- $\mathrm{C}_{10} \mathrm{H}_{18}$ ]; 1,3-dimethyladamahtane [1,3-DMA], 1,3,5trimethyladamahtane [1,3,5-TMA] and 1-ethyladamahtane [1-EA] 
Cyclic hydrocarbons and perfluorocarbons. The absolute entropies obtained by the third thermodynamic law from the experimental data and statistical thermodynamics for some cyclic and bicyclic hydrocarbons and perfluorocarbons are listed in Table 8 . The values of the absolute entropies determined by independent methods agree within errors limits from (0.1 to 1$) \%$ that proves their mutual conformity and also the reliability of the all experimental data used for computing the value of $S_{m}^{O}(g)($ expt $)$, namely the heat capacities, enthalpies of vaporization, and saturation vapor pressures.

Ferrocene derivatives [FD]. The experimental data on thermodynamics of the phase equilibriums for FD are very scarce. Therefore, critical analysis of the available data on thermodynamic properties and using them for science prognosis of failing properties are the urgent problems. A verification of reliability and mutual congruence of the properties of some derivatives of ferrocene were carried out on the basis of absolute entropies, which were computed by the third thermodynamic law from the experimental data and by statistical thermodynamics and group equation method. Comprising the $S_{m}^{O}$ and $C_{p, m}^{\circ}$ values determined by both theoretical methods showed that they agree within errors limits, $\leq 1.2 \%$. The values of absolute entropies in homologous series of alkyl- and acyl-ferrocenes obtained in this work and available in the literature are presented in Table 9.

\begin{tabular}{|c|c|c|c|c|}
\hline Compound & $S_{m}^{O}(\text { cond })^{a}$ & $S_{m}^{O}(g)(\operatorname{expt})^{a}$ & $S_{m}^{O}(g)(\text { calc })^{b}$ & $S_{m}^{o}(g)(\text { recom })^{c}$ \\
\hline $\mathrm{F}(\mathrm{cr})$ & $211.85 \pm 1.1^{d}$ & $361.0 \pm 3.6$ & $362.6 \pm 7$ & $362 \pm 3.1$ \\
\hline $\mathrm{EF}(\mathrm{liq})$ & $(354.1 \pm 1.1)^{e}$ & $(525.3 \pm 2.9)^{e}$ & $453 \pm 7 d$ & $453 \pm 7$ \\
\hline n-PF (liq) & $356.9 \pm 0.9$ & $494.3 \pm 5.0$ & $489 \pm 7$ & $492 \pm 6$ \\
\hline$i$-BF (liq) & $377.6 \pm 1.9$ & $517.3 \pm 5.2$ & $513 \pm 7$ & $515 \pm 6$ \\
\hline$n$-BF (liq) & $398.1 \pm 1.2^{e}$ & $(586.3 \pm 3.4)^{e}$ & $527 \pm 7$ & $527 \pm 7$ \\
\hline $\mathrm{FF}(\mathrm{cr})$ & $241.3 \pm 0.7^{e}$ & $(398.1 \pm 2.8)^{e}$ & $435 \pm 7 d$ & $435 \pm 7$ \\
\hline $\mathrm{AF}(\mathrm{cr})$ & $264.6 \pm 0.8$ & $478.3 \pm 2.9$ & - & $478 \pm 3$ \\
\hline POF (cr) & $291.0 \pm 1.2$ & $502.7 \pm 5.1$ & $513 \pm 7$ & $507 \pm 6$ \\
\hline$i$-BTF (liq) & $379.6 \pm 1.2$ & - & $541 \pm 7$ & $541 \pm 7$ \\
\hline
\end{tabular}

Table 9. The absolute entropies $\left(\mathrm{J} \cdot \mathrm{K}^{-1} \cdot \mathrm{mol}^{-1}\right)$ in condensed and ideal gas states of ferrocene, $[\mathrm{F}]$, and some derivatives of ferrocene: ethylferrocene $[\mathrm{EF}], n$-propylferrocene $[n-\mathrm{PF}]$, isobutylferrocene $[i$-BF], $n$-butylferrocene $[n-\mathrm{BF}]$, formylferrocene $[\mathrm{FF}]$, acetylferrocene $[\mathrm{AF}]$, propionylferrocene $[\mathrm{POF}]$, iso-butyrylferrocene $[i-\mathrm{BTF}]$ at $T=298.15 \mathrm{~K}$

The $S_{m}^{O}(g)($ expt $)$ and $S_{m}^{O}(g)($ calc $)$ values for ferrocene, $n$-propyl- and iso-butylferrocene agree to within their errors that proves the reliability of these values and all the thermodynamic data used for their calculation. The mutual consistency of the absolute entropies of iso-butyrylferrocene and iso-butylferrocene in the liquid state was revealed using the Benson additive method. The difference between the entropies of these compounds, $\Delta S=2 \mathrm{~J} \cdot \mathrm{K}^{-1} \cdot \mathrm{mol}^{-1}$, is close to the entropy increment $\Delta^{\prime} S=1.4 \mathrm{~J}^{\prime} \mathrm{K}^{-1} \mathrm{~mol}^{-1}$, which fit for replacing the $\mathrm{CH}_{2}$ by $\mathrm{CO}$ group in the passage from iso-butyl- to iso-butyrylferrocene.

The difference between the $S_{m}^{O}(g)($ expt $)$ and $S_{m}^{O}(g)($ calc $)$ values for crystal propionylferrocene, which exceeds measurement errors, can be explained by uncertainties 

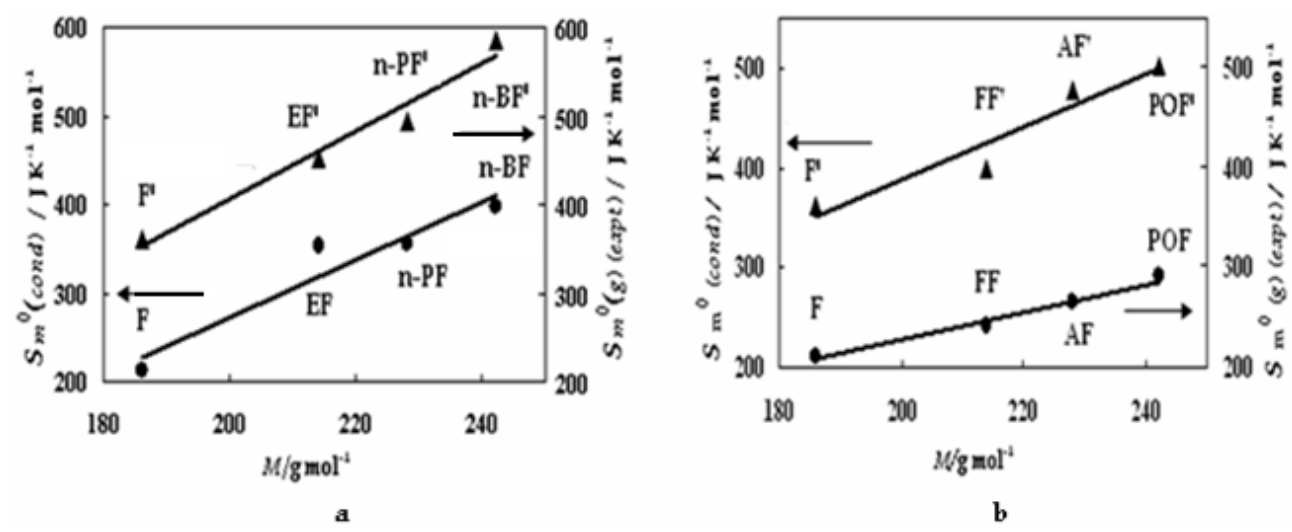

Fig. 13. Absolute entropies $\left(\mathrm{J} \cdot \mathrm{K}^{-1} \cdot \mathrm{mol}^{-1}\right)$ as functions of molecular weights $(M)$ at $T=298.15$ $\mathrm{K}$ for (a) series of alkylferrocenes in condensed $(\mathrm{F}, n-\mathrm{PF}$, and $n-\mathrm{BF})$ and ideal gas states $\left(\mathrm{F}^{\prime}\right.$, $\mathrm{EF}^{\prime}, n-\mathrm{PF}^{\prime}$, and $\left.n-\mathrm{BF}^{\prime}\right)$ where straight lines correspond to equations: $S_{m}^{o}($ cond,$A l F)=$ 3.250. $M-377.1$ and $S_{m}^{O}(g, A l F)=3.840 \cdot M-361.8$; and $(\mathbf{b})$ series of acylferrocenes in the condensed ( $\mathrm{F}, \mathrm{FF}, \mathrm{AF}$, and $\mathrm{POF})$ and ideal gas states $\left(\mathrm{F}^{\prime}, \mathrm{FF}^{\prime}, \mathrm{AF}^{\prime}\right.$, and $\left.\mathrm{POF}^{\prime}\right)$ where the straight lines correspond to equations: $S_{m}^{O}($ cond,$A c F)=1.392 \cdot M-50.68$ and $S_{m}^{O}(g, A c F)=$ 2.664. $M-144.6$

of the entropy of vapor compression calculation over the liquid phase because there are no vapor pressure data over the crystals. The verification of reliability of the other $S_{m}^{o}(g)($ expt $)$ values was analyzed on the basis of the additivity principle for extensive properties in homologous series of compounds.

As is seen from Table 9, the discrepancies between $S_{m}^{o}(g)($ expt $)$ and $S_{m}^{O}(g)$ values of EF, $n$ $\mathrm{BF}$, and FF are 5-10 times larger than the errors of these values. To find the reasons for these discrepancies, the linear correlations between the absolute entropies and molecular weights of the compounds were analyzed in homologous series of alkyl-, $S_{m}^{O}(A l F)$, and acyl-, $S_{m}^{O}(A c F)$, derivatives of ferrocene (Fig. 13).

The root-mean-square deviation [RMS] of the calculated $S_{m}^{o}($ cond, AlF) values from experimental ones was $\sigma_{S}=29 \mathrm{~J} \cdot \mathrm{K}^{-1} \mathrm{~mol}^{-1}$. Besides, the entropy of ethylferrocene deviates by the value of $10.5 \%$ from the linear dependence of the other homologues. These deviations are several times larger than the uncertainty of $S_{m}^{o}$ (cond) values and can be explained by errors in calorimetric heat capacities, obtained in (Karyakin, et al., 2003). After excluding of this value, the coefficient $R^{2}$ of linear correlation increased from 0.9147 to 0.9992, and the $\sigma_{S}$ value decreased to $4 \mathrm{~J}^{\mathrm{K}} \cdot \mathrm{K}^{-1} \mathrm{~mol}^{-1}$. Thus, the value $S_{m}^{O}($ cond $)$ for EF is unreliable. The $S_{m}^{O}(g)($ expt $)$ entropies for $n$-propylferrocene and $n$-butylferrocene deviate from the smoothed straight line (Fig. 13) by only (3.9 and 3.2) \%, respectively, nevertheless the RMS deviation of calculated $S_{m}^{o}(g)($ expt $)$ values from initial ones $\sigma_{S}=29 \mathrm{~J} \cdot \mathrm{K}^{-1} \cdot \mathrm{mol}^{-1}$ is fairly large. After replacing the absolute entropy of $n-\mathrm{BF}$, obtained from experimental data, on the $S_{m}^{O}(g)($ calc $)$ value, calculated by the empirical method of group equations, the $\sigma_{S}$ deviation 


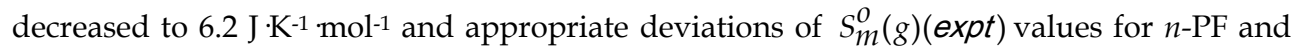
$n$-BF from the smoothed straight line did not exceed $\sim 1 \%$.

Correlations for the absolute entropies of acylferrocenes obtained for the condensed and ideal gas states (Table 9) are shown in Fig. 13, b. The RMS deviation $\sigma_{S}=6 \mathrm{~J} \cdot \mathrm{K}^{-1} \mathrm{~mol}^{-1}$ of the experimental $S_{m}^{O}(c o n d, A c F)$ entropies from the smoothed straight line conforms to the errors of these values. Appropriate deviation for the ideal gas entropies $S_{m}^{O}($ expt $A c F)$ is substantially larger, $\sigma_{S}=23.4 \mathrm{~J}^{-} \mathrm{K}^{-1} \mathrm{~mol}^{-1}$. Besides, the deviation of the entropy of formylferrocene (Table 9) from smoothed straight line, $27.5 \mathrm{~J} \cdot \mathrm{K}^{-1} \mathrm{~mol}^{-1}$, is $\sim 10$ times larger than the experimental error, which can be explained by errors in vapor pressure and the entropies of sublimation for FF. After replacing this $S_{m}^{O}(g)($ expt $)$ value by the $S_{m}^{O}(g)($ calc $)$ entropy obtained by the method of statistical thermodynamics (Table 9), appropriate deviations of the ideal gas entropies from smoothed $S_{m}^{O}(g, A c F)$ line decreased to $\leq 1.4 \%$ for the all compounds of the $A c F$ - series. This deviation is close to the measurement errors, which indicate to unreliability of the $S_{m}^{O}(g)($ expt $)$ value for FF.

A critical analysis of the absolute entropies of the alkyl and acyl derivatives of ferrocene shows that the $S_{m}^{O}(g)($ calc $)$ and $S_{m}^{O}(g)($ expt $)$ values for $n$-propylferrocene, isobutylferrocene, acetylferrocene, propionylferrocene, and iso-butyrylferocene are reliable to within errors. The entropies of ethylferrocene in the condensed state and $n$-butylferrocene and formylferrocene in the ideal gas states are not reliable because of errors in EF heat capacity measurements and errors of the vapor pressures and enthalpy of vaporization and sublimation for $n$-BF and FF, respectively. On the basis of the critical analyses of the absolute entropies of the ferrocene derivatives, the recommended reliable $S_{m}^{o}(g)($ recom $)$ values have been presented in Table 9.

\subsection{Extrapolation of vapor pressure to entire range of liquid phase}

A saturation vapor pressure of substances for entire liquid region was obtained by calculation methods on the basis of the experimental $p T$ data of moderate "atmospheric range" from 2 to $101.6 \mathrm{kPa}$. Extending the $p T$ parameters to the region of low pressures is performed by simultaneous processing the vapor pressure and differences between the heat capacities of the ideal gas and liquid. The vapor pressures above $100 \mathrm{kPa}$ are calculated by the empirical bimodal equation obtained by processing the $p T$ parameters and densities of liquids using the one-parameter corresponding states law in Filippov's version.

The extrapolation of vapor pressure to the low-temperature region down to the triple point is carried out by a system of two equations:

$$
\left\{\begin{array}{l}
\ln (p /<p>)=A^{\prime}+B^{\prime} / T+C^{\prime} \cdot \ln T+D^{\prime} \cdot T \\
\left(\Delta C_{\mathrm{p}, \mathrm{m}} / 2\right) / R=(1 / 2) \cdot\left\{C_{\mathrm{p}, \mathrm{m}}^{0}(\mathrm{~g})-C_{\mathrm{p}, \mathrm{m}}^{0}(\mathrm{liq})\right\} \cdot R=C^{\prime} / 2+D^{\prime} \cdot T
\end{array}\right.
$$

where $\langle p\rangle$ is the vapor pressure at the average temperature of experimental range, and $A^{\prime}$, $B^{\prime}, C^{\prime}$, and $D^{\prime}$ are coefficients of simultaneous processing of the $p T$ data with $\Delta C_{p, m}$ differences.

Mathematical processing by a system of equations (23) requires the use of heat capacities of the ideal gas and liquid in the neighbourhood of the triple point, where the linear temperature dependence of $C_{p, m}$ is valid. 
The method of extrapolation of the vapor pressure was tested with standard substances 1,1,1-trifluoro-2,2-dichloroethane and $n$-decane for which precision $p T$ parameters and heat capacities of the ideal gas and liquid are available in a wide temperature range.

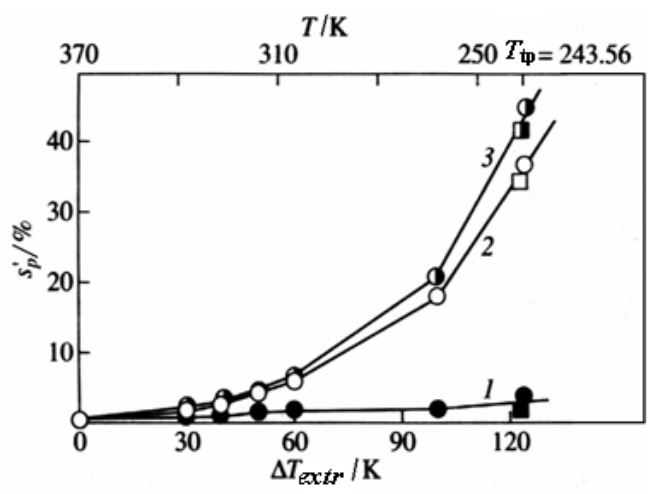

Fig. 14. Deviation $s_{p}^{\prime}=\left\{\left(p_{\text {recom }}-p_{\text {extr }}\right) / p_{\text {recom }}\right\} \times 100$ of extrapolated values $p_{\text {extr }}$ of the vapor pressure of $n$-decane from experimental recommended values $p_{\text {recom }}$ as functions of temperature range of extrapolation $\Delta T_{\text {extr }}: 1$ - calculation by a system of equations (23); 2 and 3 - calculation by equation (4) using full and shortened $(\Delta T=50 \mathrm{~K})$ interval of $p T$ parameters

Fig. 14 presents comparison of the extrapolation capabilities of equations (4) and the system of equations (23) by processing the precision $p T$ data of $n$-decane in moderate range of pressure and the $\Delta C_{p, m}$ differences in the neighbourhood of triple point $T_{t p}=243.56 \mathrm{~K}$. The deviations of the extrapolated $p$ values from the recommended experimental vapor pressures of $n$-decane (Boublik et al., 1984) and most reliable calculated values (Ruzicka \& Majer, 1994) were evaluated using equation (4) (curves 2 and 3) and the system of equations (23) (curve 1). Curves 2 and 3 were obtained for the complete and shortened temperature ranges of the $p T$ data $\Delta T=(80$ and 50$) \mathrm{K}$, respectively. In so doing, the deviation of the calculated $p$ values from experimental ones, $s_{p}^{\prime}$, increase for equation (4) from (5 to 40$) \%$ in dependence on the temperature range of extrapolation, $\Delta T_{\text {extr }}$, from (50 to 120$) \mathrm{K}$. The appropriate deviations for the system of equations (23) are much smaller and equal from (2 to 4$) \%$ for this range of extrapolation.

The results of approximation of $p T$ data for 1,1,1-trifluoro-2,2-dichloroethane (Weber, 1992) by individual equation (4) and by system of equations (23) are compared in Table 10 . The data were processed by the least squares method using the vapor pressure in the temperature range from 256 to $299 \mathrm{~K}$ and the heat capacities of the ideal gas (Frenkel et al., 1994) and liquid (Varushchenko et al., 2007) in the range from 150 to $240 \mathrm{~K}$.

In so doing, the temperature range of extrapolation of vapor pressure from initial temperature $256 \mathrm{~K}$ to $T_{t p}=145.68 \mathrm{~K}$ equals $\Delta T_{\text {extr }}=111 \mathrm{~K}$. Tentative errors of approximation coefficients were evaluated only for revealing the change of extrapolation prediction in going from equation (4) to that of (23). As is seen from Table 10, errors of 
coefficients of equations (23) are approximately ten times lower than those of equation (4), which explains increasing the extrapolation capabilities of the system (23). The resultant value of the vapor pressure at triple point $p_{t p}$ is about $25 \%$ lower than that of equation (4). Reducing the temperature range of initial $p T$ data, $\Delta T$, from (25 to 50) $\mathrm{K}$ (accordingly, extending the range of extrapolation of vapor pressure to $\sim 130 \mathrm{~K}$ ) leads to the variation of $p_{t p}$ value by only $4-6 \%$.

\begin{tabular}{|c|c|c|c|c|c|}
\hline Equation & $A / A^{\prime}$ & $-\left(B / B^{\prime}\right)$ & $-\left(C / C^{\prime}\right)$ & $\left(D / D^{\prime}\right) \times 10^{-3}$ & $p_{t p}, \mathrm{~Pa}$ \\
\hline$(4)$ & $\begin{array}{c}98.82786 \pm 11.09 \\
(10 \%)\end{array}$ & $\begin{array}{c}6061.251 \pm 273.0 \\
(4 \%)\end{array}$ & $\begin{array}{c}14.58016 \pm 1.97 \\
(10 \%)\end{array}$ & $\begin{array}{c}17.7902 \pm 3.56 \\
(20 \%)\end{array}$ & 0.155 \\
\hline$(23)$ & $\begin{array}{c}78.44263 \pm 0.496 \\
(0.6 \%)\end{array}$ & $\begin{array}{c}5677.915 \pm 29.87 \\
(0.5 \%)\end{array}$ & $\begin{array}{c}10.76986 \pm 0.093 \\
(1 \%)\end{array}$ & $\begin{array}{c}9.0292 \pm 0.23 \\
(2.5 \%)\end{array}$ & 0.114 \\
\hline
\end{tabular}

Table 10. The coefficients of equations (4) and (23) and saturated vapor pressure of 1,1,1trifluoro-2,2-dichloroethane at the triple point, $T_{t p}=145.68 \pm 0.02 \mathrm{~K}$ (Weber, 1992)

Analysis of calculated data for $n$-decane and 1,1,1-trifluoro-2,2-dichloroethane revealed that the use of the system of equations (23) for extrapolation of the vapor pressure in a wide temperature range $\Delta T_{\text {extr }} \leq\left(120\right.$ to130) $\mathrm{K}$ enabled one to obtain $p\left(T_{t p}\right)$ values with errors of $\leq 10 \%$.

Extending the saturation vapor pressure to the critical region and computing the critical parameters of the substances were performed by the corresponding states law of Filippov's version (Filippov, 1988). A large class of normal (unassociated) liquids obey the oneparameter law of corresponding states [OLCS] which manifests an existence of the oneparameter family of $\pi-\varphi-\tau$ surfaces of liquids. For the saturation curve, the OLCS equation has the form $\pi=\pi\left(\tau, A^{\prime}\right)=0$ or $\varphi=\varphi\left(\tau, A^{\prime}\right)$, where $A^{\prime}$ is the similarity criterion. The latter is assigned by ordinate $\pi$ with abscissa $\tau=0.625$ on the reduced curve of saturated vapor pressure, $A_{F}^{\prime}=100 \pi$ at $\tau=0.625$.

Few literature data are available for critical parameters of organic compounds. The law of corresponding states in Filippov's version enables one both to obtain the required critical parameters using precise $p T$ data and density $\rho$ of liquid and to calculate numerous thermophysical properties on the basis of known values of $T_{C}, V_{\mathcal{C}}$, and $A_{F}^{\prime}$. Generalized equations for the calculation of the critical parameters and of the thermophysical properties were obtained by empirical method using the array of experimental data on the appropriate properties for studied compounds (Filippov, 1988). The critical parameters were calculated by algorithms (Filippov, 1988) and (Varushchenko et. al., 1987), in which the following pairs of input data were used:

$$
\left|\begin{array}{ll}
T_{n b .} & p=101.325 \\
T_{2} & p_{2} \\
T_{3} & \rho
\end{array}\right| \text { or }\left|\begin{array}{ll}
T_{n b .} & p=101.325 \\
T_{2} & d \ln (p) / d(1 / T) \\
T_{3} & \rho
\end{array}\right|
$$


The derivative $d \ln (p) / d(1 / T)$ was determined by differentiation of the equations $\ln (p)=F(T)$ for investigated substances. A random sampling of 14 compounds, having reliable data on the vapor pressure, density, and critical parameters was used to demonstrate (Varushchenko et. al., 1987) that 1) the employed algorithm is capable to produce adequate results in computing $T_{\mathcal{C}}, V_{\mathcal{C}}$, and $A_{F}^{\prime}$ and 2) the calculation algorithm originally developed for compounds with the similarity criterion $1 \leq A_{F}^{\prime} \leq 4$ is suitable for a wider class of compounds, including polyatomic molecules with the criterion $0.5 \leq A_{F}^{\prime} \leq 4$. A prediction of the thermophysical properties by the OLCS of Filippov (Filippov, 1988) requires following basic quantities: $T_{\mathcal{C}}, V_{\mathcal{C}}$, and $A_{F}^{\prime}$. The errors of the latter calculation are within $\pm 1-2 \%$; the appropriate errors of $P_{\mathcal{C}}$, are higher and amount to $\pm 3-5 \%$.

The saturation vapor pressure are extrapolated to the critical region using the empirical binodal equation with pseudocritical parameters $T^{*} \geq T_{\mathcal{C}}$ and $P^{*} \geq P_{\mathcal{C}}$ :

$$
\log \left(P^{*} / p\right)=a \cdot \log \left(T^{*} / T\right)+\left[T^{*} / T-1\right] \cdot\left[b+c\left(T^{*} / T\right],\right.
$$

where $a=3.9726, b=0.3252$, and $c=0.40529$.

Two-parameter formula (25) weakly depends on similarity criterion $A_{F}^{\prime}$; therefore, it may be employed for curvilinear extrapolation of saturated vapor pressure using only two pairs of the $p T$ data. The formula was derived by the similarity conversion, namely using superposition of curves $-\log (\pi) /-\log (\tau)$ which have the same slope for different substances. As a result, the family of curves reduces to a single curve which has segments of vapor pressure in the supercritical region. The pair of the $P^{*}-T^{*}$ parameters is of importance for remote point on the binodal curve, which enables one to calculate the vapor pressure of a substance in the region from the normal boiling temperature to the critical point. The error of the pressure calculation is $\leq(3$ to 5$) \%$ depending on the temperature range of $p T$ data extending and their reliabi lity.

Therefore, analysis of equations (4) and (23) demonstrated that equation (4) obtained by approximation of vapor pressure of the "atmospheric range" gives precision results when it used as an interpolation equation. It is further employed for extrapolation of the vapor pressure in the temperature range of $\leq 50 \mathrm{~K}$ with an error of (1 to 2$) \%$. Extending the vapor pressure to the entire liquid phase from the critical to the triple points temperatures is performed by means of one-parameter law of corresponding states with errors from (3 to 5) $\%$ and, respectively, by simultaneous processing of the $p T$ parameters and the differences between low-temperature heat capacities $\Delta C_{p, m}=C_{p, m}^{0}(g)-C_{p, m}^{0}($ liq $)$ with uncertainties of $\leq 10 \%$, respectively. The data on extrapolation of the vapor pressure are suitable for many technological calculations.

\section{Conclusion}

The fundamental thermodynamic investigations of the phase equilibriums of many functional organic compounds were carried out by experimental and calculation methods in Luginin's Thermochemistry Laboratory of the Moscow State University. Modified setups have been created for precise determination of the saturation vapor pressures by 
comparative ebulliometry, the enthalpies of vaporization by evaporation calorimetry, and the low temperature heat capacity and phase transitions by vacuum adiabatic calorimetry. The saturation vapor pressures were determined in moderate range of pressure $2 \leq(\mathrm{p} / \mathrm{kPa})$ $\leq 101.6$ with accuracy of the temperature $\pm \leq 0.01 \mathrm{~K}$, and pressure, $\pm \leq 26 \mathrm{~Pa}$, which correspond to the modern precision levels. The temperature dependences of the saturation vapor pressure, $\ln (p)=F(T)$, and the enthalpies of vaporization $\Delta H_{\text {vap }}=f(T)$ were obtained by mathematical processing of the $p T$ parameters by equation (1), derived on the basis of Clapeyron equation using LSM with orthogonal functions. The latter allow one to calculate the errors of $\Delta H_{\text {vap }}$ values, which are urgent problem because the indirect method is the main source for determination of the enthalpies of vaporization. An agreement of the $\Delta H_{\text {vap }}$ values obtained by direct (calorimetric) and indirect (calculation) methods proves their reliability.

The precise saturated vapor pressure data are extended to entire region of the liquids under study. Extrapolation of the $p T$ parameters down to the triple point temperature are carried out by simultaneous processing the vapor pressures and low-temperature differences $\Delta C_{p, m}=C_{p, m}^{0}(g)-C_{p, m}^{0}($ liq $)$, which are the second derivatives of the vapor pressure upon the temperature. Extending the $p T$ parameters to the critical region and calculation of the critical quantities are performed by Filippov's one-parameter law of the corresponding states. The latter enables us both to calculate the critical parameters on the basis of more readily available $p T$-data and density of liquids and to predict numerous thermo-physical properties of the equilibrium liquid - vapor by means of the known critical quantities $T_{\mathcal{C}}, V_{\mathcal{C}}$, and criterion of similarity $A_{F}^{\prime}$.

The low temperature heat capacity in the temperature region (5 to 373 ) K, molecular motion in crystal and metastable phases, and solid state transitions and fusion were investigated by the adiabatic calorimetry. Uncertainties of the $C_{p, m}$ measurements are on the average $\sim 0.2 \%$ which correspond up-to-date precision level.

An accurate calorimetric study of the solid states of the functional organic compounds revealed different polymorphic modifications of the molecules, order - disorder transitions involving orientational and conformational disorder, glass-like transitions, and plastic crystalline phases with anisotropic and isotropic reorientations of the molecules. For interpretation of these transformations, the X-ray crystallography, infrared and Raman molecular spectroscopy were got involved in investigation.

The main thermodynamic functions in three aggregate states: the absolute entropy by the $3^{\mathrm{d}}$ law of thermodynamics, the changes of the enthalpy and free Gibbs energy are derived on the basis of the heat capacity and vapor pressure measurements. A critical analysis and verification of the reliability of obtained data are very significant parts of the thermodynamic investigation. With this in mind, the experimental thermodynamic functions are compared with calculated ones by additive principles and by statistical thermodynamics coupled with quantum mechanical (QM) calculation on the basis of DFT method. The QM calculation are performed on the level B3LYP/6-31G $(\mathrm{d}, \mathrm{p})$ by Gaussian 98 and 03 software packages.

A qualitative analysis of thermodynamic properties in dependence on some parameters responsible for intermolecular interactions and short range order of the liquid phase has been carried out for verification of the mutual consistency of the properties in homologous series and the series of the same type of compounds. A quantitative verification of the 
properties were performed by comparison of the absolute entropies, obtained by the third thermodynamic law, $S_{m}^{o}(g)($ expt $)$, and calculated by statistical thermodynamics, $S_{m}^{o}($ stat $)$. The agreement between these quantities confirms their reliability, as well as all experimental and calculation data used in computing process.

\section{Acknowledgment}

We are grateful to Professor O. Dorofeeva for providing Gaussian programs and assistance in quantum-chemical calculations of the ideal gas thermodynamic functions. Many thanks to Professor S. Verevkin for helping in determination of the vapor pressures of some derivatives of ferrocene and Dr. L. Pashchenko for taking part in determination of the vapor pressures of some freons. Special thanks are to Post-graduate student E. Tkachenko for providing illustrative materials of the chapter.

This work was financially supported by Russian Foundation for Basic Research under Grants No. 96-02-05445 and No. 05-02-17435.

\section{Appendix}

\subsection{Mathematical processing of saturation vapor pressure}

The vapor pressures in dependence on temperature are treated by a relationship:

$$
\begin{gathered}
-R T \ln (p)=\Delta H_{\langle T}-\alpha_{1} \cdot T+\alpha_{2} \cdot\{T-\langle T\rangle-T \cdot(\ln \mathrm{T}-\ln \langle\mathrm{T}\rangle)\} \\
\quad+\alpha_{3} \cdot\left[T^{2} / 2-\langle T\rangle^{2} / 2-T \cdot\langle T\rangle \cdot(\ln T-\ln \langle T\rangle)\right]
\end{gathered}
$$

where $\left.\alpha_{1}=\left(\Delta H^{\prime}\right\rangle^{\prime} / T\right\rangle+R \ln \left(p_{\langle T}\right)$.

Coefficients of equation (A1) are calculated by LSM using orthogonal functions (Kornilov \& Vidavski, 1969).

Two functions $\varphi_{k}$ and $\varphi_{s}$ are orthogonal if the following condition is satisfied:

$$
\left[\varphi_{k} \varphi_{S}\right]=0 \text { at } k \neq s
$$

According to Gauss, the $\sum$ sign was replaced by []. If there are $m$ functions $\Phi_{0}, \Phi_{1}, \ldots \Phi_{m}$ they may be replaced by linear combination of the orthogonal functions $\varphi_{0}, \varphi_{1}, \ldots \varphi_{p}$ using Shmidt method:

$$
\varphi_{0}=\Phi_{0} ; \varphi_{1}=\Phi_{1}-\varepsilon_{10} \varphi_{0} ; \varphi_{2}=\Phi_{2}-\varepsilon_{20} \varphi_{0}-\varepsilon_{21} \varphi_{1} ; \ldots \varphi_{p}=\Phi_{p}-\sum_{q=0}^{q=p-1} \varepsilon_{p q} \varphi_{q} \ldots,
$$

where coefficients of orthogonality, $\varepsilon_{p q}$, are defined from equation (A3) and the orthogonal condition as: 


$$
\varepsilon_{p q}=\left[\Phi_{p} \varphi_{q}\right] /\left[\varphi_{q}^{2}\right]
$$

A description of the technique of vapor pressures approximation by equation (A1) is given bellow.

The data on the saturation vapor pressure in dependence on the temperature have unequal accuracy; therefore treating those by the LSM were carried out using statistical weights $w_{i}$ :

$$
\left\{-R T \ln \left(p_{i}\right)\right\} \cdot w_{i}=\Phi\left(T_{i}\right)
$$

The value of $w_{i}$ is introduced by inversely proportional to the dispersion:

$$
w_{i}=1 /\left\{2 \cdot s\left[-R T_{i} \ln \left(p_{i}\right)\right]\right\}^{2}
$$

In conformity with the law of an accumulation of the random errors, the dispersions were calculated from the formula:

$$
s^{2}\left\{-R T_{i} \ln \left(p_{i}\right)\right\}=\left\{-R \ln \left(p_{i}\right)\right\}^{2} s_{T}^{2}+\left\{-R T_{i} / p_{i}\right\}^{2} s_{p}^{2}
$$

For simplification of the calculation, equation (A1) is reduced to the linear form:

$$
Y=a_{0}+a_{1} x_{1}+a_{2} x_{2}+a_{3} x_{3}
$$

where $\left.\left.Y=-R T \ln (p), \quad a_{0}=\Delta H^{\prime}\right\rangle^{\prime}, \quad a_{1}=-\left\{\Delta H^{\prime}\right\rangle_{T} /\left\langle T+R \ln (p\rangle_{T}\right)\right\}, \quad a_{2}=\alpha_{2}$ ， $a_{3}=-\alpha_{3}, \quad x_{1}=T$,

$$
x_{2}=\left\{T-\langle T\rangle-T[\ln (T)-\ln (\langle T)]\}, \quad x_{3}=\left\{T^{2} / 2-\langle T\rangle^{2} / 2-T\langle T\rangle[\ln (T)-\ln (T\rangle)\right]\right\} .
$$

Owing to transition to the orthogonal functions, equation (A7) takes a form:

$$
Y=b_{0} \varphi_{0}+b_{1} \varphi_{1}+b_{2} \varphi_{2}+b_{3} \varphi_{3}
$$

where $\varphi_{0}=1, \quad \varphi_{1}=x_{1}-\varepsilon_{10} \varphi_{0}, \quad \varphi_{2}=x_{2}-\varepsilon_{20} \varphi_{0}-\varepsilon_{21} \varphi_{1}$, $\varphi_{3}=x_{3}-\varepsilon_{30} \varphi_{0}-\varepsilon_{31} \varphi_{1}-\varepsilon_{32} \varphi_{2}$.

The coefficients of orthogonality, $\varepsilon_{p q}$ (A4), expressed with the use of statistical weights, are:

$$
\begin{gathered}
\varepsilon_{10}=\left[x_{1} \varphi_{0} w\right] /\left[\varphi_{0}^{2} w\right], \quad \varepsilon_{20}=\left[x_{2} \varphi_{0} w\right] /\left[\varphi_{0}^{2} w\right], \quad \varepsilon_{30}=\left[x_{3} \varphi_{0} w\right] /\left[\varphi_{0}^{2} w\right] \\
\varepsilon_{21}=\left[x_{2} \varphi_{1} w\right] /\left[\varphi_{1}^{2} w\right] \\
\varepsilon_{31}=\left[x_{3} \varphi_{1} w\right] /\left[\varphi_{1}^{2} w\right], \quad \varepsilon_{32}=\left[x_{3} \varphi_{2} w\right] /\left[\varphi_{2}^{2} w\right]
\end{gathered}
$$


The coefficients $b_{p}$ of equation (A8) were determined by LSM using a minimum of a sum of the deviations square of the experimental $y_{i}=-\left\{R T \ln \left(p_{i}\right)\right\} w_{i}$ values from those ones calculated by (A8):

$$
\sum_{i}\left[y_{i}-\left(b_{0} \varphi_{0}+b_{1} \varphi_{1 i}+b_{2} \varphi_{2 i}+b_{3} \varphi_{3 i}\right)\right]^{2}=\min
$$

By differentiation of the expression (A10) upon $b_{p}$ and followed by transformation of the obtained equations using a ratio (A2), a system of normal equations were obtained, every equation being contained only one unknown coefficient:

$$
\begin{gathered}
{\left[y_{i} \varphi_{0} w_{i}\right]-b_{0}\left[\varphi_{0}^{2} w_{i}\right]=0} \\
{\left[y_{i} \varphi_{1} w_{i}\right]-b_{1}\left[\varphi_{1}^{2} w_{i}\right]=0} \\
{\left[y_{i} \varphi_{2} w_{i}\right]-b_{2}\left[\varphi_{2}^{2} w_{i}\right]=0} \\
{\left[y_{i} \varphi_{3} w_{i}\right]-b_{3}\left[\varphi_{3}^{2} w_{i}\right]=0}
\end{gathered}
$$

Thus, the system of the normal equations is a diagonal matrix. The formulae intended for calculation of $b_{p}$ coefficients were obtained on the basis of the normal system of equations (A11):

$$
\begin{gathered}
b_{0}=\left[y_{i} \varphi_{0} w_{i}\right] /\left[\varphi_{0}^{2} w_{i}\right], \quad b_{1}=\left[y_{i} \varphi_{1} w_{i}\right] /\left[\varphi_{1}^{2} w_{i}\right], b_{2}=\left[y_{i} \varphi_{2} w_{i}\right] /\left[\varphi_{2}^{2} w_{i}\right], \\
b_{3}=\left[y_{i} \varphi_{3} w_{i}\right] /\left[\varphi_{3}^{2} w_{i}\right] \\
b_{p}=\left[y_{i} \varphi_{p} w_{i}\right] /\left[\varphi_{p}^{2} w_{i}\right]
\end{gathered}
$$

The $s^{2}\left(b_{p}\right)$ dispersions of the coefficients $b_{p}$ were defined in accordance with the formula (A12), as:

$$
s^{2}\left(b_{p}\right)=s_{0}^{2}\left(\partial b_{p} / \partial y\right)^{2}=s_{0}^{2} /\left[\varphi_{p}^{2} w_{i}\right]
$$

where $s_{0}^{2}=r^{-1} \sum_{i}\left(y_{i}-Y_{i}\right)^{2}$ is dispersion of individual measurement, $r=n-l$ denotes a number of a freedom degree, $n$ is a number of measurements, $l$ is the number of terms of approximation equation, $y_{i}$ are experimental values of the $y(x)$ function, $Y_{i}$ are the values of the function calculated from approximated equation.

The constants $a_{i}$ of equation (A1) were calculated after the coefficients of $b_{p}$ and $\varepsilon_{p q}$ have been rounded off. The $b_{p}$ and $\varepsilon_{p q}$ coefficients were rounded up to the values of their uncertainties, but one spare digit being excepted. The errors of $b_{p}$ and $\varepsilon_{p q}$ coefficients were calculated by formulae: 


$$
\Delta b_{p}=t_{0.05}\left\{s_{0}^{2} /\left[\varphi_{p}^{2} w\right]\right\}^{1 / 2} \text { and } \Delta \varepsilon_{p q}=\Delta x_{p} /\left\{\left[\varphi_{p}^{2} w\right]\right\}^{1 / 2},
$$

where $\Delta x_{p}$ is uncertainty of rounding of initial $x_{p}$ value. Dispersion of $s_{0}^{2}$ value and sums $\left[\varphi_{p}{ }^{w}\right]$, which were used by computing the $\Delta b_{p}, \Delta \varepsilon_{p q}$, and $b_{i}$ constants (A12) were rounded off up to three significant figures. On the basis of formula (A7) and uncertainty of the temperature measurements of $\pm 0.006 \mathrm{~K}$, the $\Delta x_{i}$ errors were evaluated equal to: $\Delta x_{1}=$ $0.006 \mathrm{~K}, \Delta x_{2}=0.024 \mathrm{~K}$ and $\Delta x_{3}=0.048 \mathrm{~K}^{2}$.

The constants $a_{1}, a_{2}$, and $a_{3}$ are easily determined by means of coefficients of $b_{p}$ and $\varepsilon_{p q}$ as $\varphi$-functions are linear combination of the $x_{1}, x_{2}$, and $x_{3}$ variable values:

$$
a_{3}=b_{3}, \quad a_{2}=b_{2}-\varepsilon_{32} b_{3}, \quad a_{1}=b_{1}-\varepsilon_{21} b_{2}-\varepsilon_{31} b_{3}+\varepsilon_{32} \varepsilon_{21} b_{3}
$$

The constant $a_{0}$ of equation (A7) are determined when replacing its terms by appropriate mean ones, namely $\langle x\rangle_{i w}$ and $\langle y\rangle_{w}$ :

$$
a_{0}=\left\langle y_{w}\right\rangle-a_{1}\left\langle x_{1 w}\right\rangle-a_{2}\left\langle x_{2 w}\right\rangle-a_{3}\left\langle x_{3 w}\right\rangle
$$

A calculation of the $a_{p}$ coefficients by the method described above is simpler than straight treating the $p T$-parameter by equation (A7) using LSM. For decreasing a number of tabulated coefficients, the final equation (A7) was transformed to the form:

$$
\ln (p)=A+B / T+C \cdot \ln (T)+D \cdot T
$$

Here $A, B, C$, and $D$ are correlated constants, which were obtained by reducing the similar terms of equation (A7). A number of significant digits in the coefficients were chosen based on a condition: deviations of calculated vapor pressures from the experimental ones should not exceed the uncertainties of determination of the $p$ values $(13 \div 26 \mathrm{~Pa})$. Then, the accuracy of each coefficient of equation (A16) was designated depending on the accuracy of $\ln (p)=f(T)$ function using derivatives $\partial \ln (p) / \partial K$, where $K=A, B, C$ or $D$ coefficients. If $\partial \ln (p) / \partial K \approx \Delta \ln (p) / \Delta K=X$, then $\Delta K=\Delta \ln (p) / X$. This formula was used for evaluation of $\Delta K$ values on the basis maximum values of $p=101.6 \mathrm{kPa}$ and $T=530 \mathrm{~K}$, determined in modified ebulliometer.

$$
\begin{aligned}
& \Delta A=\Delta \ln (p)=\frac{\Delta p}{p}=\frac{13 \cdot 10^{-3}}{101.6}=0.00010 \\
& \Delta B=T \cdot \Delta \ln (p)=\frac{T \cdot \Delta p}{p}=\frac{530 \cdot 13 \cdot 10^{-3}}{101.6}=0.07 \\
& \Delta C=\frac{\Delta \ln (p)}{\ln (T)}=\frac{\Delta p}{p \cdot \ln (T)}=\frac{13 \cdot 10^{-3}}{101.6 \cdot 6.3}=0.00002 \\
& \Delta D=\frac{\Delta \ln (p)}{T}=\frac{\Delta p}{p \cdot T}=\frac{13 \cdot 10^{-3}}{101.6 \cdot 530}=0.0000002
\end{aligned}
$$




\subsection{Calculation of the enthalpies of vaporization}

Mathematical processing the saturated vapor pressure in dependence on temperature by LSM with orthogonal functions makes it possible to calculate both the enthalpy of vaporization and its dispersion $s^{2}\left(\Delta_{v a p} H\right)$. The latter for the thermodynamic values $F=\Delta H_{T}$ is designated based on the law of an accumulation of the random errors by the formula:

$$
s^{2}(F)=s_{0}^{2} \Sigma\left(\partial F / \partial y_{i}\right)^{2}
$$

An equation for computing the temperature dependence of the enthalpy of vaporization was obtained by means of formula (A1) and Clapeyron equation (2) (part 2.1) taking account on interconnections between the $\alpha_{0}, \alpha_{2}, \alpha_{3}$ constants of (A7) and (A15) and connections of these coefficients with $b_{p}$ by (A14), respectively:

$$
\begin{aligned}
& \left.\Delta H_{T}^{\prime}=\Delta H_{\langle T}^{\prime}\right\rangle \alpha_{2}\left(T-\langle T\rangle+\alpha_{3}\left(T^{2}-\langle T\rangle^{2}\right) / 2=\left\langle y_{w}\right\rangle-b_{1}\left\langle x_{1 w}\right\rangle+b_{2}\left(\varepsilon_{21}\left\langle x_{1 w}\right\rangle+T-\langle T\rangle-\left\langle x_{2 w}\right\rangle+\right.\right. \\
& \left.+b_{3}\left\{\left(\varepsilon_{31}-\varepsilon_{32} \varepsilon_{21}\right)\left\langle x_{1 w}\right\rangle+\varepsilon_{32}(T\rangle+\left\langle x_{2 w}\right\rangle-\langle T\rangle^{2} / 2-\left\langle x_{3 w}\right\rangle-\varepsilon_{32} T+T^{2} / 2\right)\right\}
\end{aligned}
$$

Computing the dispersion, $s^{2}\left(\Delta H_{T}^{\prime}\right)$, caused by errors of the $p T$-data, was carried out by the formula, deduced based on (A17):

$$
\begin{gathered}
s^{2}\left(\Delta H_{T}^{\prime}\right)=s_{0}^{2} \sum_{i}\left\{\left(\partial \Delta H_{T}^{\prime}\right) /\left(\partial y_{i}\right)\right\}^{2}=s_{0}^{2}\left\{\frac{1}{\left[w_{i}\right]}+\frac{\left\langle x_{1 w}\right\rangle^{2}}{\left[\varphi_{1}^{2} w_{i}\right]}+\frac{(T+\gamma)^{2}}{\left[\varphi_{2}^{2} w_{i}\right]}+\frac{\left(\lambda-\varepsilon_{32} T+T^{2} / 2\right)^{2}}{\left[\varphi_{3}^{2} w_{i}\right]},\right. \\
\text { where } \gamma=\varepsilon_{21}\langle T\rangle-\left\langle x_{2 w}\right\rangle-\langle T\rangle, \\
\lambda=\left(\varepsilon_{31}-\varepsilon_{32} \varepsilon_{21}\right)\left\langle x_{1 w}\right\rangle+\varepsilon_{32}\left(\langle T\rangle+\left\langle x_{2 w}\right\rangle\right)-\langle T\rangle^{2} / 2-\left\langle x_{3 w}\right\rangle
\end{gathered}
$$

According to approximation accepted by deriving of the equation (A6), the enthalpy of vaporization, $\Delta_{\text {vap }} H$, equals to product of the $\left(\Delta H_{T}^{\prime}\right)$ and $\Delta Z$ values:

$$
\Delta_{v a p} H=\left\{\Delta H^{\prime}\langle T\rangle+\alpha_{2}(T-\langle T\rangle)+\alpha_{3}(T-\langle T\rangle)^{2} / 2\right\} \cdot \Delta Z,
$$

where $\Delta Z$ is the difference of the compressibility factors, taking into account vapor deviation from ideality and volume changes of the vapor and liquid. For decreasing a number of tabulated coefficients, the equation (A19) was transformed to the form:

$$
\Delta_{\text {vap }} H=R\left(-B+C T+D T^{2}\right) \cdot \Delta Z
$$

The coefficients of equation (A20) are connected with correlations: 


$$
B=\left(\alpha_{2}\langle T\rangle+\alpha_{3}\langle T\rangle^{2} / 2-\Delta H^{\prime}\langle T\rangle\right) / R, \quad C=\alpha_{2} / R, \quad \text { and } \quad D=\alpha_{3} / 2 R .
$$

As an example, Table A1 lists the parameters of equation for calculating the uncertainties of the enthalpies of vaporization caused by errors of the vapor pressures for some freons.

\begin{tabular}{|c|c|c|c|c|c|c|c|c|c|}
\hline Compounds & $t_{0.05}$ & $a$ & $b$ & $-c$ & $d \cdot 10^{-3}$ & $-e$ & $g$ & $\begin{array}{c}s\left\{\Delta H_{m}^{\prime}(T)\right\} \\
\mathrm{J} \cdot \mathrm{mol}^{-1}\end{array}$ & $\begin{array}{c}\left.s\left\{\Delta H_{m}^{\prime}\right) / \Delta H_{m}^{\prime}\right\} \cdot 10^{2}, \\
\%\end{array}$ \\
\hline $\mathrm{CFCl}_{2} \mathrm{CFCl}_{2}$ & 2.306 & 0.389 & 0.0104 & 339 & 0.66 & 56918 & 337.6 & 43.1 & 0.12 \\
\hline $\mathrm{CF}_{2} \mathrm{ClCFCl}_{2}$ & 2.775 & 114 & 21.64 & 308 & - & - & - & 130.6 & 0.45 \\
\hline $\mathrm{CF}_{2} \mathrm{BrCF}_{2} \mathrm{Br}$ & 2.569 & 27.8 & 3.709 & 310 & - & - & - & 60.2 & 0.21 \\
\hline
\end{tabular}

Table A1. Parameters of equation for calculation of the $s\left\{\Delta H_{m}^{\prime}(T)\right\}$ values by

$$
s\left\{\Delta H_{m}^{\prime}(T)\right\} /\left(J \cdot \mathrm{mol}^{-1}\right)= \pm{ }_{0.05} \cdot\left[a+b \cdot\left\{(T / K+c\}^{2}+d \cdot\left\{e+g \cdot(T / K)-0.5 \cdot(T / K)^{2}\right\}^{2}\right]^{1 / 2}\right.
$$

for freons $\mathrm{CFCl}_{2} \mathrm{CFCl}_{2}, \mathrm{CF}_{2} \mathrm{ClCFCl}_{2}$ and $\mathrm{CF}_{2} \mathrm{BrCF}_{2} \mathrm{Br}$ at $T=298.15 \mathrm{~K}$, where $t_{0.05}$ denotes the Student criterion.

This equation was obtained by summing up dispersions of the orthogonal coefficients of equation (A19). Errors of the $\Delta_{v a p} H_{m}(T)$ values caused by uncertainties of $p T$ data equal to those of the direct calorimetric methods (Table 2).

\section{References}

Adachi K.; Suga H. \& Seki S. (1968). Phase changes in crystalline and glassy-crystalline cyclohexanol. Bulletin Chemical Society of Japan, 41, No 5, (May 1968), p.p.1073-1087, ISSN 0009-2673

Alexandrov, Yu.I. (1975). Tochnaja Kriometrija Organicheskih Veschestv (Exact criometric of organic substances). Khimiya, Leningrad

Boublik, T.; Fried, V. \& Hala, E. (1984). The vapour pressure of pure substances, Elsevier, ISBN 0-444-41097-X, Amsterdam

Chikos, J.S. \& Acree Jr., W.E. (2003). Enthalpies of vaporization of organic and organometallic compounds. Journal of Physical and Chemical Reference Data, Vol. 32, No 2, p.p. 519-878, ISSN 0047-2689

Cohen, N. \& Benson, S.W. (1993). Estimation of Heat of Formation of Organic Compounds by Additivity Methods. Chemical Reviews. Vol. 93, (1993), p.p. 2419-2438

Domalski, E.S. \& Hearing, E.D. (1993). Estimation of the Thermodynamic Properties of C-HN-O-S-Halogen Compounds at 298.15 K. Journal of Physical and Chemical Reference Data, Vol. 22, No 4, p.p. 805-1159, ISSN 0047-2689

Emel'yanenko, V.N.; Verevkin, S.P.; Krol, O.V.; Varushchenko, R.M. \& Chelovskaya, N.V. (2007). Vapour pressures and enthalpies of vaporization of a series of the ferrocene derivatives. Journal of Chemical Thermodynamics., Vol. 39, No 4, (April 2007), p.p. 594-601, ISSN 0021-9614

Emel'yanenko, V.N.; Krol, O.V.; Varushchenko, R.M.; Druzhinina, A.I. \& Verevkin, S.P. (2010). The Termodynamic Characteristics of Ferrocene Alkyl and Acyl Derivatives. 
Russian Journal of Physical Chemistry A. Vol. 84, No 7, (July 2010), p.p. 1089-1097, ISSN 0036-0244

Filippov, L.P. (1988). Metodi rascheta i prognozirovaniya svoistv veschestv. (Methods of calculation and prediction of properties of substances). Moscow State University, ISBN 5-211-00096-X, Moscow

Frenkel, M.; Kabo, G.J.; Marsh, K.N.; Roganov, G.N. \& Wilhoit, R.C. (1994). Thermodynamics of Organic Compounds in the Gas State, Thermodynamics Research Center, College Station, ISBN 1-883400-03-1 (Vol.1), ISBN 1-883400-05-8 (Vol.2), Texas, USA

Frisch, M.J.; Trucks, G.W.; Schlegel, H.B.; et al., (2003). GAUSSIAN 03, Revision B.03, Gaussian, Inc, Pittsburgh, PA

Higgins, E.R. \& Lielmers, J. (1965). Thermodynamics Functions for Freon 113. Journal of Chemical and Engineering Data. Vol. 10, No 2, (April 1965), p.p. 178-179, ISSN 00219568

Karyakin, N. V.; Kozlova, M. S.; Sheiman, M. S.; Kamelova, G.P. \& Larina, V.N. (2003). Thermodynamic functions of derivatives of ferrocene. Russian Journal of Physical Chemistry A. Vol. 77, No 8, (August 2010), p.p. 1230-1236, ISSN 0036-0244

Kolesov, V.P. (1995). The order - disorder transitions in some organic crystals. Thermochimica Acta, Vol. 266, (November 1995), p.p. 129-145, ISSN 0040-6031

Kornilov, A.N. \& Vidavski, L.M. (1969). Some questions of statistical processing of the thermodynamic data. III. Approximation of dependence $\Delta G=\Delta H-T \cdot \Delta S$. Zh. Fiz. Khim. Vol. 43, No 9, (September 1969), p.p. 2224- 2230, ISSN 0044-4537

Kulikov, D.V; Verevkin, S.P. \& Heintz, A. (2001). Enthalpies of Vaporization of a Series of Aliphatic Alcohols. Experimental Results and Values Predicted by the ERASModel. International Journal Fluid Phase Equilibria. Vol. 192, No 1-2, (Desember 2001), p.p. 187-207, ISSN 0378-3812

Lawson, D.D.; Moacanin, J.; Scherer Jr., K.V; Terranova, T.F. \& Ingham, J.D. (1978). Methods for the estimation of vapor pressures and oxygen solubility of fluorochemicals for possible application in artificial blood formulations. Journal of Fluorine Chemistry. Vol. 12, No 3, (September 1978) p.p. 221-236, ISSN 0022-1139

Majer, V. \& Svoboda, V. (1985). Enthalpies of vaporization of organic compounds: A Critical Review and Data Compilation. IUPAC. Blackwall Science, ISBN 0632015292, Oxford

NIST Chemistry Webbook. Standard Reference Data. http://webbook.nist.gov/chemistry

Poling, B.E.; Prausnitz, J.M. \& O'Connell, J.P. (2001). The Properties of Gases and Liquids. 5-th Edition, McGrow-Hill, ISBN 0-07-011682-2 (Errata at http:/ / www.che.virginia.edu/PGL5 , (2010))

Pvese, F. \& Malishev, V.M. (1994). Routine measurements of specific heat capacity and thermal conductivity of high- $T_{\mathrm{c}}$ superconducting materials in the range $4-300 \mathrm{~K}$ using modular equipment. Advances in Cryogenic Engineering, Vol. 40, Proceedings of the 1993 International Cryogenic Materials Conference (ICMC), Albuquerque, New Mexico,p. p. 119-124, ISBN 0-306-44823-8

Ries J.G. (1991). Fluorocarbon - Based in vivo Oxygen Transport and Delivery Sistem. Vox Sanquinis. Vol. 61, No 4, (December 1991), p.p. 225-239, ISSN 1423-0410

Ruzicka, K. \& Majer, V. (1994). Simultaneous Treatment of Vapour Pressures and Related Thermal Data between the Triple and Normal Boiling Temperatures for n-Alkanes $\mathrm{C}_{5}-\mathrm{C}_{20}$. Journal of Physical and Chemical Reference Data, Vol. 23, No 1, p.p. 1-39, ISSN 0047-2689 
Sabbe, M.K.; De Vleeschouwer, F.; Reyniers M.-F.; Waroquier M. \& Marin G.B. (2008). First Principles Based Group Additive Values for the Gas Phase Standard Entropy and Heat Capacity of Hydrocarbons and Hydrocarbon Radicals. Journal of Physical Chemistry, A, Vol. 112, No 47, (November 2008), p.p. 12235-12251, ISSN 1089-5639

Van Speybroeck, V.; Van Neck, D.; Waroquier, M.; Wauters, S.; Saeys, M. \& Marin G.B. (2000). Ab Initio Study of Radical Addition Reactions: Addition of a Primary Ethylbenzene Radical to Ethene (1). Journal of Physical Chemistry, A, Vol. 104, No 46, (November 2008), p.p. 10939-10950, ISSN 1089-5639

Vansteenkiste, P.; Van Speybroeck, V.; Marin G.B. \& Waroquier, M. (2003). Ab Initio Calculation of Entropy and Heat Capacity of Gas-Phase n-Alkanes Using Internal Rotations. Journal of Physical Chemistry, A, Vol. 107, No 17, (April 2003), p.p. 31393145, ISSN 1089-5639

Van Wijk, M.F.; \& Smit W.M. (1960) Impurity determination by thermal analysis. I. The melting curve of gradually frozen sample. Analytica Chimica Acta. Vol. 23, No 6 (December 1960), p.p. 545-551, ISSN 0003-2670

Varushchenko, R.M.; Halchenko, G.L. \& Medvedev, V.A. (1977). Precision calorimetric device for measurement enthalpy of evaporation. Zh. Fiz. Khim. Vol. 51, No 4, (April 1977), p.p. 992-996, ISSN 0044-4537

Varushchenko, R. M.; Druzhinina, A. I. \& Pashchenko, L. L. (1987). Vapor pressure and critical parameters dichloroalkanes, Zh. Fiz. Khim. Vol. 61, No 9, (September 1987), p.p. 2327-2332, ISSN 0044-4537

Varouchtchenko, R.M. \& Droujinina, A.I. (1995). Thermodynamics of vaporization of some perfluorotrialkilfmines. Journal of Chemical Thermodynamics., Vol. 27, No 4, (April 1995), p.p. 355-368, ISSN 0021-9614

Varushchenko, R. M.; Druzhinina, A. I. \& Sorkin, E. L. (1997a). Low-temperature heat capacity of 1-bromoperfluoro- octane. Journal of Chemical Thermodynamics., Vol. 29, No 6, (June 1997), p.p. 623-637, ISSN 0021-9614

Varushchenko, R. M.; Druzhinina, A. I. \& Korshunova, M. V. (1997b). Low-temperature heat capacities and thermodynamic properties of 1,1-difluoro-1,2,2-trichloroethane and 1,2-difluoro-1,1,2-trichloroethane. Journal of Chemical Thermodynamics., Vol. 29, No 10, (October 1997), p.p. 1059-1070, ISSN 0021-9614

Varushchenko, R.M.; Druzhinina, A.I.; Kuramshina, G.M. \& Dorofeeva, O.V. (2007). Thermodynamics of vaporization of some freons and halogenated ethanes and propanes. International Journal Fluid Phase Equilibria. Vol. 256, No 1-2, (August 2007), p.p. 112-122, ISSN 0378-3812

Wadsö, I. (1966). A Heat of Vaporization Calorimeter for Work at $25{ }^{\circ} \mathrm{C}$ and for Small Amounts of Substances. Acta Chemica Scandinavica Vol. 20, No 2, (February 1966), p.p. 536-543, ISSN 0904-213X

Weber, L.A. (1992). Ebulliometric Measurement of the Vapor Pressures of R123 and R141b. International Journal Fluid Phase Equilibria. Vol. 80, (November1992), p.p. 141-148, ISSN 0378-3812

Westrum, E.F. \& McCullough, J.P. (1965). Thermodynamics of crystals, In: Physics and Chemistry of the Organic Solid State. Eds.: Fox, D., Labes, M.M. \& Weissberger, A., Vol. 1, p.p. 5-155, Interscience, New-York - London 


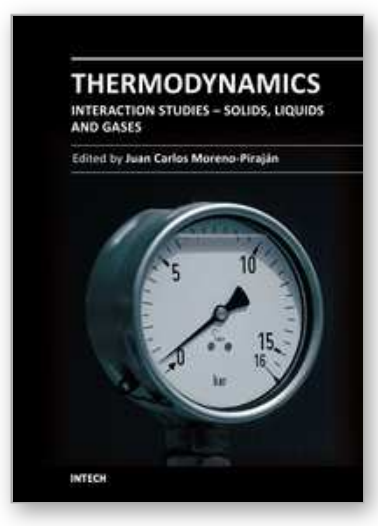

\author{
Thermodynamics - Interaction Studies - Solids, Liquids and Gases \\ Edited by Dr. Juan Carlos Moreno Pirajễin
}

ISBN 978-953-307-563-1

Hard cover, 918 pages

Publisher InTech

Published online 02, November, 2011

Published in print edition November, 2011

Thermodynamics is one of the most exciting branches of physical chemistry which has greatly contributed to the modern science. Being concentrated on a wide range of applications of thermodynamics, this book gathers a series of contributions by the finest scientists in the world, gathered in an orderly manner. It can be used in post-graduate courses for students and as a reference book, as it is written in a language pleasing to the reader. It can also serve as a reference material for researchers to whom the thermodynamics is one of the area of interest.

\title{
How to reference
}

In order to correctly reference this scholarly work, feel free to copy and paste the following:

Raisa Varushchenko and Anna Druzhinina (2011). Thermodynamics of the Phase Equilibriums of Some Organic Compounds, Thermodynamics - Interaction Studies - Solids, Liquids and Gases, Dr. Juan Carlos Moreno PirajÃ $i n(E d)$, ISBN: 978-953-307-563-1, InTech, Available from:

http://www.intechopen.com/books/thermodynamics-interaction-studies-solids-liquids-andgases/thermodynamics-of-the-phase-equilibriums-of-some-organic-compounds

\section{INTECH}

open science | open minds

\section{InTech Europe}

University Campus STeP Ri

Slavka Krautzeka 83/A

51000 Rijeka, Croatia

Phone: +385 (51) 770447

Fax: +385 (51) 686166

www.intechopen.com

\section{InTech China}

Unit 405, Office Block, Hotel Equatorial Shanghai

No.65, Yan An Road (West), Shanghai, 200040, China

中国上海市延安西路65号上海国际贵都大饭店办公楼 405 单元

Phone: +86-21-62489820

Fax: $+86-21-62489821$ 
(C) 2011 The Author(s). Licensee IntechOpen. This is an open access article distributed under the terms of the Creative Commons Attribution 3.0 License, which permits unrestricted use, distribution, and reproduction in any medium, provided the original work is properly cited. 